\title{
12. REWORKED BENTHIC FORAMINIFERS FROM SITE 802, EAST MARIANA BASIN, WESTERN EQUATORIAL PACIFIC ${ }^{1}$
}

\author{
Winton G. Wightman ${ }^{2}$
}

\begin{abstract}
Reworked shallow-water larger and deep-water calcareous benthic foraminifers were recovered from foraminiferal packstones and nannofossil chalks in Hole 802A. The autochthonous zeolitic pelagic claystone is characterized by late Campanian abyssal agglutinated foraminifers that allow correlation with the North Atlantic and the adjacent Pigafetta Basin. Assemblages of Dendrophrya/Rhizammina in graded beds within the zeolitic claystone indicate reworking through entrainment in the flocculent E layer of turbidites, rather than recolonization following a biosiliceous event. Background sedimentation of the claystone took place below the carbonate compensation depth.

The nannofossil chalk contains reworked lower bathyal to abyssal calcareous foraminifers of late Paleocene to early Miocene age. The topmost bed of the nannofossil chalk unit commences with an algal foraminiferal packstone containing Lepidocyclina sumatrensis, Heterostegina borneensis, Amphistegina hauerina, Asterigerina marshallana, and A. tentoria, which indicate that the source area was a shallow-water reef and allow the bed to be dated as early Miocene. The absence of obviously younger planktonic microfossils in the graded bed indicates that the resedimentation event was generally contemporaneous with original deposition and took place during an early Miocene global sea-level highstand.

An early Miocene shallow-water assemblage is also seen in the graded beds at the base of a volcaniclastic turbidite sequence overlying the nannofossil chalks. Resedimentation of this unit was associated with volcanic activity some distance away.
\end{abstract}

\section{INTRODUCTION AND GEOLOGICAL BACKGROUND}

Ocean Drilling Program (ODP) Hole $802 \mathrm{~A}$ was drilled at $12^{\circ} 5.778^{\prime} \mathrm{N}$, $153^{\circ} 12.6258^{\prime} \mathrm{E}$, in a water depth of $6968 \mathrm{~m}$ in the central part of the East Mariana Basin in the western equatorial Pacific, between Japan and Australia (Fig. 1). The East Mariana Basin is a relatively flat abyssal basin, bordered to the north by the Magellan Seamounts and to the south by additional seamounts including the Ita Mai Tai guyot and the Caroline Islands. The location of Site 802 southeast of a magnetic lineation sequence identified as M22 to M31 suggested that it was underlain by Upper Jurassic basement, and the main objectives at Site 802 were to sample Jurassic sediments and oceanic crust in order to study the evolution of the Pacific Ocean (Lancelot, Larson, et al., 1990). The bottom of the hole, however, penetrated extrusive basalts that underlie upper Aptian-Albian claystones, suggesting that either the basement age was younger than predicted or that a sequence of Cretaceous lava flows overlie an older (Jurassic) sedimentary section. Hole $802 \mathrm{~A}$ bottomed at $560 \mathrm{~m}$ below seafloor (mbsf), and operations were terminated when the well seismic tool became stuck in the hole, requiring the logging cable to be severed.

The $509 \mathrm{~m}$ Cretaceous to Cenozoic sedimentary section at Hole $802 \mathrm{~A}$ was subdivided into nine lithologic units by the shipboard scientific party (Fig. 2). The section commences with a sequence of Cenomanian to upper Aptian-Albian brown claystone, calcareous claystone, radiolarian limestone, and volcaniclastic turbidites (Units VI-IX) overlain by volcaniclastic turbidites with clay, claystone, silty claystone, porcellanite, and debris flows of Coniacian to Campanian age (Unit V). Immediately above this is upper Campanian to lower Paleocene zeolitic pelagic claystone and calcareous nannofossil silty claystone with minor porcellanite, chert, and calcareous siltstone or sandstone (Unit IV). Graded beds, cross lamination, and flame structures identify the coarse-grained deposits in lower part of Unit IV as turbidites (Lancelot, Larson, et al., 1990). The overlying upper Paleocene to lower Miocene sequence consists of a $92.5-\mathrm{m}$ interval of

\footnotetext{
'Larson, R. L., Lancelot, Y., et al., 1992. Proc. ODP, Sci. Results, 129: College Station, TX (Ocean Drilling Program).

${ }^{2}$ Center for Marine Geology, Dalhousie University, Halifax, Nova Scotia, B3H 3J5 Canada.
}

nannofossil chalk with foraminifers and minor amounts of interbedded nodular chert, porcellanite, claystone, and foraminiferal chalk (Unit III). Thinly laminated chalk turbidites composed of foraminifer-rich layers overlying micrite-rich layers occur toward the base of the unit. The upper part of the unit (Cores 129-802A-27R and 129$802 \mathrm{~A}-28 \mathrm{R}$ ) contains a $5.5-\mathrm{m}$ graded bed that fines upsection from grain-supported algal foraminiferal packstone to matrix-supported nannofossil chalk. The foraminiferal packstone is composed mainly of shallow-water skeletal debris (larger foraminifers, bryozoans, red algae, and echinoid fragments) and volcanic rock fragments in a nannofossil and micrite matrix. Overlying the nannofossil chalk is a thick sequence of lower Miocene to Pliocene tuff (volcaniclastic turbidites) with calcareous claystone and chalk (Subunit IIB). Toward the base of this unit is a graded tuffaceous sandstone rich in larger foraminifers and other shallow-water debris. Tuff and pelagic claystone comprise the overlying Subunit IIA. Pelagic brown clay of Neogene to Pleistocene age forms the youngest unit of the sequence (Unit I). The stratigraphic sequence seen at Site 802 differs from those at Sites 800 and 801 , where Upper Cretaceous strata are characterized by chert and the Tertiary strata are zeolitic claystone.

\section{Benthic Foraminiferal Assemblages}

Three groups of Cretaceous and Cenozoic benthic foraminifers were recovered from Site 802 (Fig. 2 and Table 1): (1) deep-water agglutinated foraminifers (DWAF), typical of outer bathyal to abyssal water depths and restricted to zeolitic pelagic claystone (Unit IV); (2) reworked deep-water calcareous benthic ("smaller") foraminifers from above the carbonate compensation depth (CCD), found within the matrix-supported fine-grained fractions of the nannofossil chalk (Unit III) and the lower part of the zeolitic pelagic claystone (Unit IV); and (3) reworked shallow-water ("larger") foraminifers, found within the grain-supported coarse layers in the upper part of the nannofossil chalk (Unit III) and tuffaceous sandstones in the lower part (Subunit IIB) of the volcaniclastic turbidites.

Because the Site 802 DWAF assemblages were recovered from pelagic claystones that show limited reworking or redeposition, they may provide us with the best evidence of paleoenvironmental conditions for this part of the East Mariana Basin during the Late Creta- 


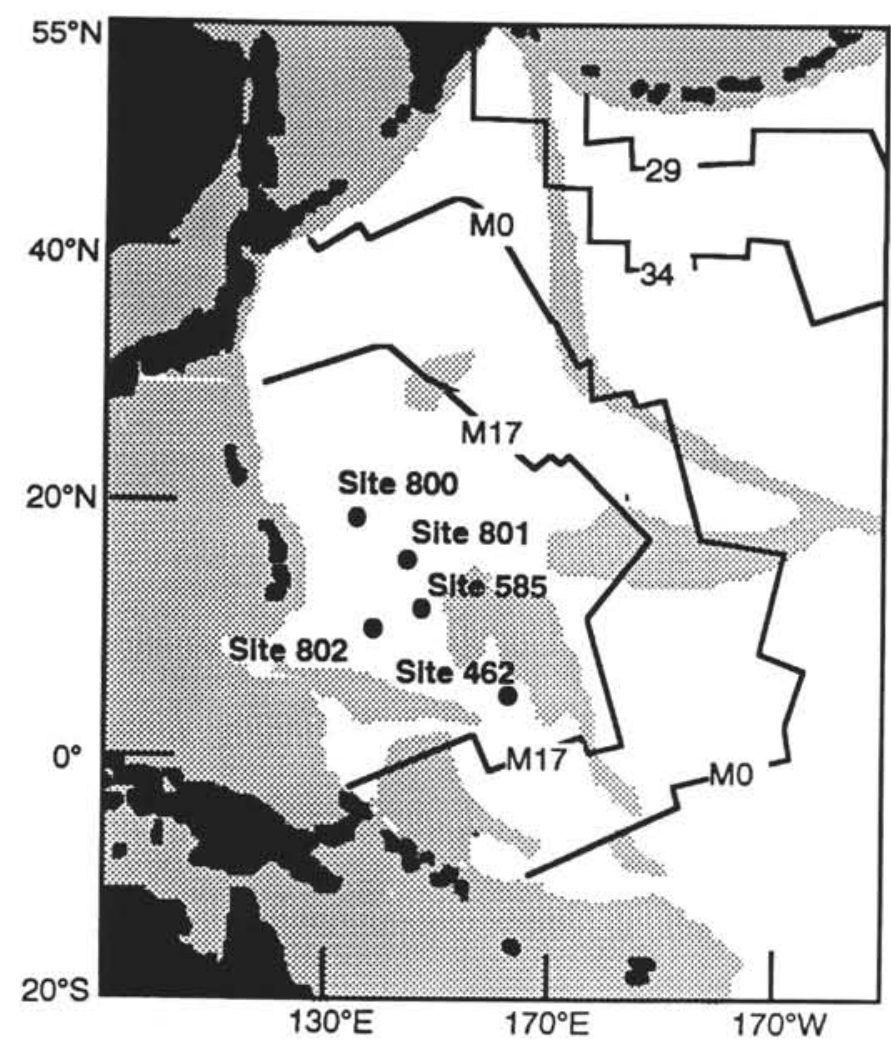

Figure 1. Location of ODP Sites 800,801 , and 802 and DSDP Sites 585 and 462 (modified from Lancelot, Larson, et al., 1990). Jagged contours represent magnetic lineations and unshaded areas represent normal Pacific oceanic crust. Shaded areas represent volcanic edifices with thickened crustal sections, as well as the younger areas beyond the Pacific subduction zones.

ceous. Study of these assemblages may also strengthen the biostratigraphic zonation already established for the western Pacific Sites 196, 198, 800, and 801 (Wightman and Kuhnt, this volume), North Atlantic Ocean (Moullade et al., 1988; Kuhnt et al., 1989), and Western Mediterranean realm (Kuhnt, 1990).

The reworked shallow-water larger foraminifers are of interest as they document erosional events through the geological history of the East Mariana Basin and provide information on the depositional setting and stratigraphy of the source environments. Deep Sea Drilling Project (DSDP) Sites 585 (East Mariana Basin) and 462 (Nauru Basin) (Fig. 1) also contained reworked benthic foraminiferal assemblages and shallowwater debris that were instrumental to the understanding of the Cretaceous and Cenozoic geological history of the region.

\section{Material and Methods}

A total of 42 samples were collected from the graded layer at the base of Unit II, the graded bed in the upper part of Unit III, the zeolitic pelagic claystone of Unit IV, and the top of the volcaniclastic turbidites (Unit V). Shipboard samples of $15-20 \mathrm{~cm}^{3}$ and core-catcher samples of about $40 \mathrm{~cm}^{3}$ were boiled in a water-Calgon solution and washed over a $63-\mu \mathrm{m}$ sieve. Shore-processed samples were kiln dried for 8-10 hr prior to washing. Samples particularly rich in foraminifers were divided into fractions using a microsplitter so as to yield about 200-300 specimens when picked. All specimens were picked and mounted in reference slides. Larger foraminifers were mounted in thermoplastic resin and ground on both sides to produce thin sections, which were photographed using an "aristophot." Other specimens were illustrated by scanning electron microscope. The distribution of species is shown in Table 1 .

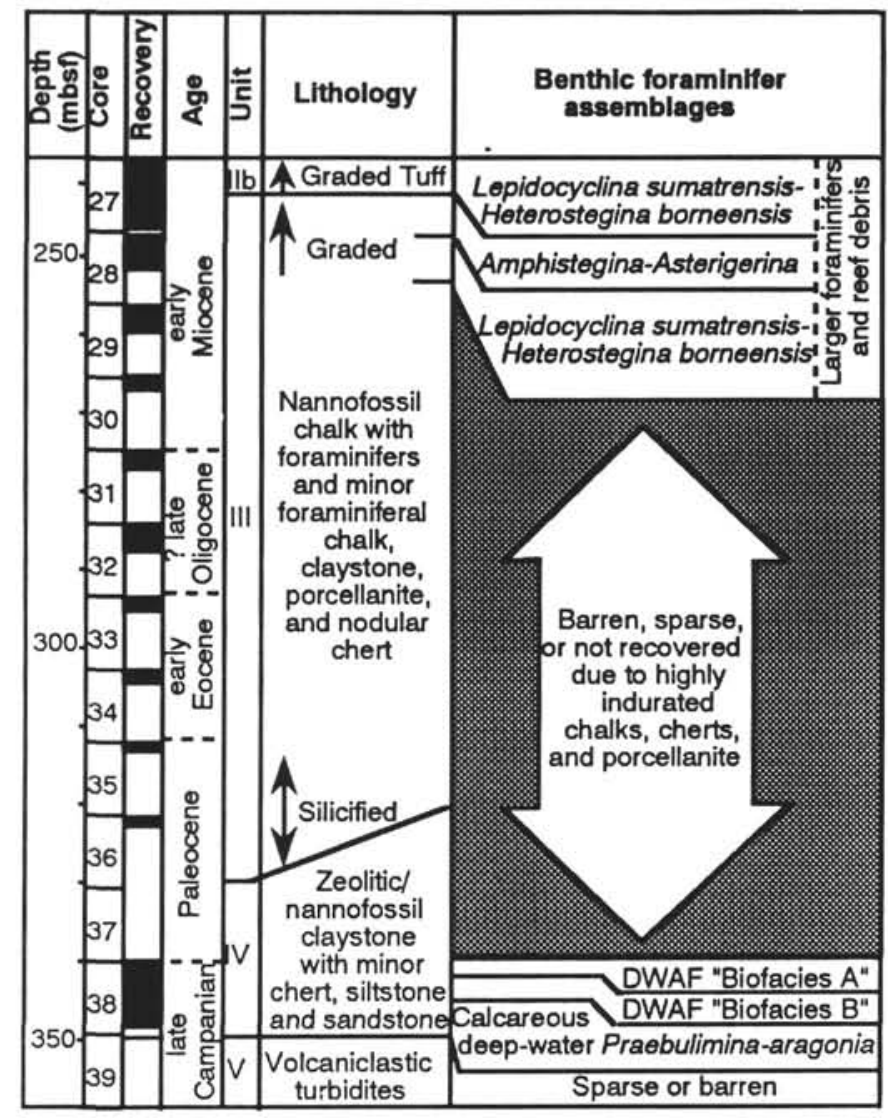

Figure 2. Lithostratigraphic section, core recovery, and benthic foraminiferal assemblages in Hole 802A. DWAF = deep-water agglutinated foraminifers.

\section{BIOSTRATIGRAPHY}

A number of stratigraphically important benthic foraminifers support the ages determined from planktonic microfossils (E. Erba in Erba and Covington, this volume). Where benthic foraminifers were absent or nondiagnostic, age determinations were made using planktonic microfossils. Reworking of the benthic foraminifers, particularly the larger shallow-water forms, does not appear to be as great a problem as it has been for the planktonic microfossils, probably because of the differences in hydrologic behavior of the two groups. A similar observation was made with respect to reworking at Site 585 (Sliter, 1986). From the limited evidence of reworking, we may assume that the resedimentation events took place at generally the same time, or soon after the time of original deposition. With the possible exception of the zeolitic pelagic claystone (Unit IV) seen in Core 129-802A-37R through Section 129-802A-39R-1, $45 \mathrm{~cm}$, the age of the youngest faunas in any part of the sequence must be considered the maximum age of the redeposited sediments.

\section{Late Campanian-?Paleocene}

Core $129-802 \mathrm{~A}-37 \mathrm{R}$, represented only by the core-catcher sample, was questionably assigned to the Paleocene on the basis of rare nannofossils. Benthic foraminifers were not seen, and poorly preserved planktonic foraminifers could not be identified to the species level.

Samples from Core 129-802A-38R are assigned a late Campanian age on the basis of a number of stratigraphically important cosmopolitan deep-water foraminifers. The calcareous benthic foraminifers Aragonia velascoensis and $A$. ouezzanensis, reported to have a Campanian first appearance (van Morkhoven et al., 1986), occur in Samples 129-802A$38 \mathrm{R}-5,54-59 \mathrm{~cm}$, and $129-802 \mathrm{~A}-38 \mathrm{R}-4,115-118 \mathrm{~cm}$. Other Late Creta- 
ceous foraminifers found in these samples include Pullenia minuta, Praebulimina cushmani, Gyroidinoides beisselli, Cibicides ribbingi, C. voltziana, Stilostomella pseudoscripta, and the agglutinant Gaudryina pyramidata, all of which have been previously reported from the Campanian-Maestrichtian of DSDP sites in the Atlantic and Pacific (Sliter, 1977, 1986).

Several stratigraphically important DWAF were also seen: Hormosina ovulum, typical of the Campanian through Maestrichtian (Moullade et al., 1988), occurs in Sample 129-802A-38R-1, 70-73 cm; Plectorecurvoides rotundus, reported from the Upper Cretaceous (Krasheninnikov, 1974), occurs in Sample 129-802A-38R-2, 48-52 cm; and other typical Late Cretaceous species include Haplophragmoides biumbilicatus, $H$. herbichi, and Praecystammina globigeriniformis.

The DWAF assemblages permit the correlation of Hole 802A with Holes 196A, 198A, 800A, and 801A in the Pigafetta Basin and the calibration of the foraminiferal zones to middle/upper Campanian strata of the North Atlantic (Fig. 3). The Haplophragmoides acme Assemblage Zone of the Pigafetta Basin, although likely ecologically controlled, is taxonomically distinct and of biostratigraphic value (Wightman and Kuhnt, this volume). Even though the ranges of most DWAF species in Hole 802A extend into the Maestrichtian in the North Atlantic, their late Campanian age at Site 802 is constrained by the overlapping ranges of the nannofossils Quadrum trifidum and Lithastrinus grillii in Sections 129-802A-38R-1, $1 \mathrm{~cm}$, through 129802A-38R-2, 29 cm (Lancelot, Larson, et al., 1990).

\section{Late Paleocene-Early Eocene}

Benthic foraminifers were absent from samples of this age range, and dating was achieved using planktonic foraminifers and nannofossils. Planktonic foraminifers including Morozovella edgari, $M$. subbotinae, M. formosa gracilis, $M$. acuta, Planorotalites chapmani, $P$. pseudomenardi, Acarinina primitiva, A. nitida, A. soldadoensis, and Subbotina sp. were found in Samples 129-802A-33R-CC and 129802A-34R-CC (Lancelot, Larson, et al., 1990), which are assigned to the $M$. edgari Zone (early Eocene). Samples 129-802A-35R-CC and $129-802 \mathrm{~A}-36 \mathrm{R}-\mathrm{CC}$ contained only poorly preserved Tertiary planktonic foraminifers. The nannofossils in these beds are of a predominantly late Paleocene assemblage, with reworked Late Cretaceous species. The uppermost Paleocene to lowermost Eocene nannofossil species, Discoaster multiradiatus, supports foraminiferal age assignments, but does not assist in locating the Paleocene/Eocene boundary.

\section{?Late Oligocene-Early Miocene}

Cores $129-802 \mathrm{~A}-27 \mathrm{R}$ to $129-802 \mathrm{~A}-32 \mathrm{R}$ are assigned an early Miocene-late Oligocene age on the basis of benthic and planktonic foraminifers and nannofossils. Of particular biostratigraphic interest are the larger foraminifer assemblages recovered from these cores. Lepidocyclina sumatrensis and Heterostegina borneensis are common along with Amphistegina hauerina, described from Miocene sediments of the Indo-Pacific region and Europe (Larsen, 1978). Amphistegina marshallana, A. tentoria, and Rotalia tectoria, reported from the lower Miocene of Bikini Island (Todd and Post, 1954), are also common. Smaller benthic foraminifers typical of Eocene through Miocene age include Bulimina tuxpamensis and Stilostomella lepidula, although according to Boltovskoy (1978), none of these are useful for determining the position of the Oligocene/Miocene boundary.

The stratigraphic ranges of Lepidocyclina sumatrensis and Heterostegina borneensis are questionable, as they have been reported from the upper Oligocene (Adams, 1960), the lower Miocene (Van der Vlerk, 1955; Cole, 1957; Coleman, 1963), and the upper Miocene (Chaproniere, 1984). Premoli Silva and Brusa (1981) also reported $L$. sumatrensis and $H$. borneensis from redeposited shallow-water debris that they regarded as late Oligocene in age at Hole 462A (Nauru Basin). Despite uncertainties concerning the ages of the latter species, the co-occurrence of Asterigerina marshallana, A. tentoria, and Rotalia tectoria in Hole 802A supports an early Miocene age, based on the ranges of these species in Bikini Atoll Drill Hole 2B (Todd and Post, 1954). On the basis of their larger foraminifer assemblages, Cores $129-802 \mathrm{~A}-27 \mathrm{R}$ and $129-802 \mathrm{~A}-28 \mathrm{R}$ correlate with early Miocene age strata in the Bikini Atoll and Eniwetok Atoll Drill Holes (Fig. 4). The nannofossil Triquetrorhabdulus carinatus, observed in Samples 129-802A-26R-CC to 129-802A-32R-CC, supports an early Miocene to late Oligocene age (Lancelot, Larson, et al., 1990).

\section{PALEOECOLOGY}

Benthic foraminiferal assemblages provide useful information about the depositional setting of the host sediment. Autochthonous faunas in abyssal clays may provide information on paleoceanographic conditions, whereas the redeposited benthic foraminiferal assemblages provide useful information about the depositional settings and stratigraphy of source environments.

\section{Depositional Setting of the Pelagic Claystone (Unit IV)}

The benthic foraminifers in the fine sandstone and siltstone turbidite layers in the lower part of Unit IV (Sections 129-802A-38R-4 through 129-802A-38R-6) are well sorted to a size fraction generally below $350 \mu \mathrm{m}$ and many specimens appear to be juveniles, resulting in a number of taxonomic uncertainties. The assemblage is characterized by the agglutinated species Gaudryina pyramidata and the calcareous benthic species Aragonia velascoensis, A. ouezzanensis, Praebulimina cushmani, Bolivina, Epistomina cf. caracolla, Cibicides ribbingi, C. voltziana, Orthomorphina, Plamulina cf. wuellerstorfi, Gyroidinoides beisseli, G. cf. subglobosus, Cassidulina, and Dentalina. Rare ostracode valves and fragments are also seen. This assemblage contains faunal elements typical of the "Nuttallides biofacies" of Tjalsma and Lohmann (1983), indicative of lower bathyal/abyssal paleobathymetries. The presence of the Early Cretaceous Epistomina cf. caracolla suggests transportation from upper bathyal or outer neritic source areas, although other shallower water indicators were not observed. The abrasion of the tests of E. caracolla supports long-distance transportation of the tests and/or aragonite dissolution.

The bathymetry of benthic species during the Late Cretaceous is poorly understood and may not be the same as has been determined for the same species for the early Tertiary; Tjalsma and Lohmann (1983) have shown that the paleobathymetries of many species changed through time, particularly during the Paleogene. The absence of autochthonous abyssal agglutinated foraminifers and the presence of calcareous bathyal assemblages in this interval indicate that these deposits represent distal turbidites.

The pelagic zeolitic claystone in the upper part of Unit IV, Sections 129-802A-38R-1 to $129-802$ A-38R-3, lacks any calcareous specimens and contains only DWAF assemblages. Two assemblage types were seen: (1) a lower diversity assemblage dominated by tubular morphotypes in Sections 129-802A-38R-2 and 129-802A-38R-3 and (2) a higher diversity assemblage of tubular and coiled morphotypes in Section 129-802-38R-1.

The lower diversity assemblage in Section 129-802-38R-3 contains specimens of DendrophryalRhizammina only. This assemblage is typical of the final stage of distal turbidite sedimentation in which the entrained E layer finally settles out, containing within it the tubular foraminifers that had previously inhabited the nepheloid layer at the sediment/water interface prior to substrate disturbance by the turbidite (Kaminski, 1988). The low diversity and dominance of tubular forms in this assemblage is similar in character to the "Biofacies B" assemblages that developed in the North Atlantic immediately following biosiliceous "events" in the Late Cretaceous (Kuhnt et al., 1989). Although similar Biofacies B assemblages are also seen at Pigafetta Basin Sites 196, 198, 800, and 801 immediately following radiolarian sedimentation (Wightman and Kuhnt, this volume), there is no evi- 
Table 1. Distribution of Cretaceous-Tertiary benthic foraminifers in Hole 802A.

\begin{tabular}{|c|c|c|c|c|c|c|c|c|c|c|c|c|c|c|}
\hline \multicolumn{15}{|l|}{ Hole 802A } \\
\hline Core, section: & $27 \mathrm{R}-1$ & $27 \mathrm{R}-1$ & $27 \mathrm{R}-2$ & $27 \mathrm{R}-2$ & $27 \mathrm{R}-2$ & $27 \mathrm{R}-3$ & $27 \mathrm{R}-3$ & $27 \mathrm{R}-4$ & $27 \mathrm{R}-5$ & $27 \mathrm{R}-6$ & 27R-CC & $28 R-1$ & 28R-1 & $28 \mathrm{R}-1$ \\
\hline Interval $(\mathrm{cm})$ : & $22-29$ & $124-129$ & $29-34$ & $60-66$ & $125-129$ & $36-40$ & $135-140$ & $110-115$ & $110-118$ & $43-47$ & & $11-15$ & $65-70$ & $100-105$ \\
\hline No. of species: & 12 & 17 & 6 & 8 & 4 & 7 & 2 & 1 & 6 & 4 & 0 & 6 & 4 & 6 \\
\hline \multicolumn{15}{|l|}{ "Larger" foraminifers } \\
\hline Amphistegina hauerina & $\mathbf{R}$ & $\mathbf{R}$ & & & A & & & . & . & . & . & . & . & . \\
\hline Asterigerina marshallana & $\mathrm{C}$ & C & C & A & 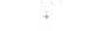 & . & & . & A & & . & . & . & $\mathrm{F}$ \\
\hline Asterigerina tentoria & . & . & . & & $\mathrm{C}$ & & & . & , & & . & . & $\therefore$ & . \\
\hline Heterostegina borneensis & $\mathrm{R}$ & $\mathbf{R}$ & $\mathrm{F}$ & - & 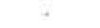 & . & . & & . & . & & & $\mathrm{F}$ & . \\
\hline Lepidocyclina sumatrensis & $\mathbf{R}$ & $\mathbf{R}$ & C & $\mathrm{F}$ & , & . & . & & . & : & & & . & + \\
\hline Paramtalia armata & : & $\mathrm{F}$ & $\therefore$ & $\mathrm{F}$ & : & & $\cdot$ & & & . & , & $\mathrm{F}$ & . & $\therefore$ \\
\hline Rotalia tectoria & $\mathrm{F}$ & $\mathrm{F}$ & A & A & A & . & . & & . & : & - & , & · & $\mathrm{F}$ \\
\hline \multicolumn{15}{|l|}{ "Smaller" foraminifers } \\
\hline Angulogerina angulosa & $\mathrm{F}$ & $\mathrm{R}$ & . & $\mathbf{R}$ & . & $\mathrm{F}$ & $\mathrm{F}$ & . & $\mathbf{F}$ & . & . & . & . & + \\
\hline Aragonia ouezzaensis & . & . & & & : & . & & - & . & . & . & . & . & + \\
\hline Aragonia velascoensis & . & & & . & . & . & & - & . & . & . & . & . & . \\
\hline Bolivina indet. spp. & $\mathrm{F}$ & $\mathbf{R}$ & F & $\therefore$ & : & $\mathrm{F}$ & . & $\mathrm{P}$ & $\mathrm{F}$ & $\mathrm{C}$ & . & : & . & $\mathrm{F}$ \\
\hline Bolivina sp. 1 & C & C & . & $\mathrm{R}$ & . & A & & $\cdot$ & C & . & . & $\mathrm{F}$ & & C \\
\hline Bulimina nuxpamensis & $\mathbf{R}$ & & . & ; & , & ; & & - & , & • & , & : & $\dot{2}$ & . \\
\hline Cassidulina subtumida & & * & . & ; & . & $\mathrm{F}$ & & $\cdot$ & & - & . & $\mathrm{F}$ & C & . \\
\hline Cibicides ribbingi & . & &. & . & . & . & . & . & . & . & . & & . & . \\
\hline Cibicides spp. & : & & . & . & $\dot{s}$ & & & $\cdot$ & ; & $\dot{5}$ & . & : & : & ; \\
\hline Cibicidoides voltriana & $\mathrm{C}$ & $\mathrm{F}$ & . & $\mathrm{F}$ & $\mathrm{C}$ & & . & · & C & C & . & A & A & A \\
\hline Dentalina spp. & . & & & & & & . & . & . & - & . & . & . & . \\
\hline Epistomina caracolla & . & . & : & & & & & . & . & $\cdot$ & *. & . & . & \\
\hline Gaudryina pyramidata & , & & . & & - & . & & . & . & * & s & & . & . \\
\hline Globorotalites michelianus & & . & & & . & & & + & & & $\cdot$ & - & . & $\cdot$ \\
\hline Gyroidinoides beisseli & . & & . & & & . & & - & . & & . & & . & \\
\hline Gyroidinoides cf. praeglobosus & . & $\mathbf{R}$ & . & & & & & - & & ? & . & . & . & . \\
\hline Lenticulina sp. 1 & . & $\mathrm{R}$ & . & & & & & . & & $\cdot$ & . & , & . & $\cdot$ \\
\hline Lenticulina spp. & $\dot{0}$ & $\therefore$ & . & & . & & & $\cdot$ & & $\dot{2}$ & . & $\therefore$ & : & : \\
\hline Loxostomum eleyi & $\mathbf{R}$ & $\mathrm{R}$ & . & . & . & $\mathrm{F}$ & $\mathrm{C}$ & $\cdot$ & . & $\mathrm{F}$ & *. & C & , & - \\
\hline Oridorsalis umbonatus & $\therefore$ & $\mathrm{F}$ & & $\mathrm{R}$ & . & & - & & $\dot{s}$ & $\dot{s}$ & . & . & . & $\dot{x}$ \\
\hline Orthomorphina aff. antillea & $\mathrm{F}$ & $\mathrm{F}$ & R & $\therefore$ & . & , & . & & C & C & - & $\mathrm{F}$ & A & $\mathrm{F}$ \\
\hline Orthomorphina ef. columnaris & - & $\mathrm{F}$ & 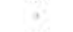 & . & $\cdot$ & : & & & . & $\therefore$ & . & $\cdot$ & . & $\cdot$ \\
\hline Plamulina cf. wuellerstorfi & . & . & . & . & . & . & & & & . & & . & . & . \\
\hline Pleurostomella cf, alternans & $\mathbf{R}$ & . & . & . & $\cdot$ & $\mathrm{F}$ & & . & , & & . & . & . & \\
\hline Praebulimina cushmani & & . & . & . & & . & . & . & . & $\cdot$ & . & . & . & . \\
\hline Pullenia mimuta & . & . & . & . & . & . & & . & . & . & . & - & . & \\
\hline Reussella cf. pseudospinulosa & . & . & . & . & : & . & . & - & . & . & . & . & . & \\
\hline Siphonina cf. tenuicarinata & & . & , & . & . & . & & . & . & - & . & - & . & \\
\hline Stilostomella lepidula & & $\mathbf{R}$ & . & & . & $\mathrm{F}$ & & $\cdot$ & & . & . & . & . & \\
\hline Stilostomella pseudoscripta & & . & . & & & & & . & & . & . & . & . & . \\
\hline Stilostomella sp. I & . & . & & & & & & $\cdot$ & & $\cdot$ & . & - & · & \\
\hline \multicolumn{15}{|l|}{ Deep-water agglutinants } \\
\hline Dendrophrya latissimalexcelsa & . & & , & . & & & . & $\cdot$ & - & - & & & . & + \\
\hline Glomospira charoides & . & & . & . & & & . & . & . & & & , & . & \\
\hline Haplophragmoides biumbilicalis & . & & & $\cdot$ & , & . & . & $\cdot$ & . & & & - & · & \\
\hline Haplophragmoides herbichi & , & * & & . & . & , & & & & $\cdot$ & .. & . & · & \\
\hline Haplophragmoides spp. & . & . & & . & . & . & . & . & & $\cdot$ & . & . & , & · \\
\hline Hormosina crassa & . & . & . & . & . & . & . & $\cdot$ & . & $\cdot$ & . & - & . & \\
\hline Hormosina ovulum & . & . & & . & . & . & . & . & . & . & . & . & . & . \\
\hline Hyperammina dilatata & . & . & ? & . & . & . & & $\cdot$ & : & . & . & - & . & $\cdot$ \\
\hline Paratrochamminoides corpulentus & . & . & . & . & . & . & * & . & . & $\cdot$ & . & , & · & : \\
\hline Paratrochamminoides intricatus & - & . & & & & . & & . & . & . & . & & $\cdot$ & \\
\hline Plectorecurvoides rotundus & & . & & & & & . & . & . & . & . & . & . & . \\
\hline Praecystammina globigerinaeformis & & & & . & & & $\cdot$ & $\cdot$ & · & & . & * & ? & : \\
\hline Rhizammina sp. 1 & . & & & & & . & & . & . & $\cdot$ & & , & · & \\
\hline Saccammina sphaerica & . & . & & & & & & $\cdot$ & . & . & & & . & . \\
\hline Subreophax scalaris & . & & & & & . & & . & . & * & & . & . & . \\
\hline Trochammina depressa & . & . & & . & . & & & . & . & & & . & . & . \\
\hline
\end{tabular}

Notes: Abundances: $\mathrm{R}=<3 \%, \mathrm{~F}=3 \%-15 \%, \mathrm{C}=16 \%-30 \%, \mathrm{~A}=>30 \%, \mathrm{P}=$ present in low numbers.

dence of a significant biosiliceous event at Site 802, and the assemblage seems more related to the final stages of turbidite deposition from Hole $802 \mathrm{~A}$.

The higher diversity assemblage in Section 129-802A-38R-1 contains species of Haplophragmoides, Hormosina, Paratrochamminoides, Hyperammina, Plectorecurvoides, Glomospira, Praecystammina, and Saccammina, in addition to DendrophryalRhizammina. Although this assemblage is of higher diversity to that seen two sections below, in comparison with assemblages seen in the North Atlantic and the Pigafetta Basin sites it is of low to moderate diversity only. This type of assemblage seems to represent the normal "background" fauna of Late Cretaceous abyssal settings. The assemblage seen in Section 129-802A-38R-2 appears to be transitional between the turbidite-influenced lower diversity assemblage and the background fauna.

\section{Foraminiferal Packstone-Nannofossil Chalk Beds (Unit III) and Volcaniclastic Turbidites (Unit II)}

The nannofossil chalk seen in Cores $129-802 \mathrm{~A}-29 \mathrm{R}$ to $129-802 \mathrm{~A}-$ $37 \mathrm{R}$ contains only sparse assemblages of small (juvenile?) calcareous benthic foraminifers that were probably derived from bathyal paleoenvironments. The high degree of induration of these beds, particularly in the lower cores, may have contributed to the poor recovery of foraminifers from this interval.

The 5.5-m graded bed that makes up the highest part of Unit III and the lowest part of Unit II contains abundant and diverse benthic foraminiferal assemblages that typically include the shallow-water "larger" foraminifers. Four main groups of larger foraminifers stand out: (1) Lepidocyclina, (2) Heterostegina, (3) the rotaliids Rotalia and Pararo- 
Table 1 (continued).

\begin{tabular}{|c|c|c|c|c|c|c|c|c|c|c|c|c|c|c|c|c|}
\hline $28 \mathrm{R}-2$ & $28 R-3$ & $28 R-3$ & 28R-3 & 28R-CC & $29 \mathrm{R}-2$ & $29 \mathrm{R}-2$ & $29 \mathrm{R}-\mathrm{CC}$ & $30 R-1$ & $30 R-1$ & 30R-CC & $3 \mid \mathrm{R}-1$ & 3IR-CC & $32 \mathrm{R}-1$ & $32 \mathrm{R}-\mathrm{CC}$ & 33R-CC & $34 \mathrm{R}-\mathrm{CC}$ \\
\hline $40-46$ & $23-28$ & $77-83$ & $117-124$ & & $2-6$ & $72-75$ & & $76-79$ & $89-91$ & & $117-120$ & & 91-95 & & & \\
\hline 9 & 5 & 13 & 9 & 12 & 4 & 6 & 0 & 0 & 0 & 0 & 0 & 1 & 1 & 0 & 0 & 0 \\
\hline $\mathrm{F}$ & C & $\mathrm{R}$ & & $\mathrm{F}$ & . & & . & & & . & & & & & . & . \\
\hline $\mathrm{F}$ & $\mathrm{F}$ & F & C & c & . & & & & & & & . & . & & & \\
\hline C & A & F & . & F & . & & . & & & & & & & & . & \\
\hline c & & F & & F & & & . & & & . & & . & & & . & \\
\hline $\mathrm{F}$ & & & C & C & & & . & & & & & . & & & . & \\
\hline , & & R & $\mathrm{R}$ & $\mathrm{R}$ & & & . & & & & & . & & & & \\
\hline C & & A & c & A & & & . & & & & & & & . & . & \\
\hline . & $\mathrm{F}$ & R & & . & . & F & & & & & & . & . & . & . & . \\
\hline & & & & & . & & . & & & . & & , & . & . & $\because$ & \\
\hline & & & & & & & $\cdot$ & & . & . & & & . & . & & \\
\hline & $\mathrm{F}$ & $\mathrm{F}$ & $\mathrm{F}$ & $\mathrm{R}$ & C & C & . & & . & & & & . & & & \\
\hline & & & F & & $\mathrm{F}$ & & . & & . & & & & . & & & . \\
\hline & & & & & & & . & & . & & & & & & & . \\
\hline C & & $R$ & $\mathrm{R}$ & R & $\mathrm{F}$ & F & . & & . & . & & . & & & & . \\
\hline . & & & & & & & & & . & - & & & & & . & . \\
\hline . & & & & & & & & & . & - & & & & & & . \\
\hline . & & $\mathrm{F}$ & $\mathrm{F}$ & F & A & A & & & . & & & $\mathrm{P}$ & P & & . & . \\
\hline . & & & & & & & & & . & . & & & & & & . \\
\hline : & & & & & & & . & & . & & & & & & & . \\
\hline . & & . & & . & & & . & & . & & & & . & . & . & \\
\hline . & & & & . & & & & . & & & & & . & . & . & \\
\hline & & & & . & & & & & & & & & . & . & . & \\
\hline & & & & . & & & & & & & & $\therefore$ & . & . & & \\
\hline & & & & $\mathrm{R}$ & & $\therefore$ & & & & & & . & . & . & & \\
\hline & & & & & & $F$ & . & & & & & & . & . & . & \\
\hline & & F & & & & . & . & & & & & & . & . & . & \\
\hline $\mathrm{F}$ & & : & R & . & & & & & & & & & . & & . & \\
\hline $\mathrm{F}$ & & $\mathrm{R}$ & & & & & & & & & & & & & . & \\
\hline & & & & & & & . & & & & & & & & & \\
\hline & & & & & & & . & & & & & & & & & \\
\hline & & R & & & & & & & & & & & & . & . & . \\
\hline & & & . & & & . & & & & & & & & & . & . \\
\hline & & & . & & & & . & & & & & . & & . & & . \\
\hline & & & & & & $R$ & & & & & & & & & & . \\
\hline & & & & & & $\because$ & . & & & & & . & . & & & \\
\hline & & & & & & & . & & . & & & . & . & . & & \\
\hline & & & & & & . & . & & . & & & . & . & . & & \\
\hline & & & & F & & . & . & & & & & & . & & & \\
\hline & & & & & & & & & & & & & & & & \\
\hline & , & & , & & & & & & & & & & & & & \\
\hline & . & & . & & & & & & & & & & & & . & . \\
\hline & . & & . & & & & & & & & & & & . & . & \\
\hline & & & . & & & . & & & & & & $\therefore$ & & . & . & \\
\hline & & & . & & & . & . & & & & & . & & . & . & \\
\hline & & & & & & . & . & & & . & & . & . & . & & \\
\hline & & & & & & . & . & & & . & & & . & & & \\
\hline & & & & & & . & & & . & . & & . & & & & \\
\hline & & & & & & & . & & & & & & & & & \\
\hline & & & . & & & & . & & & & & & & & & \\
\hline & & & & & & & . & & & & & & & . & & . \\
\hline & . & & & & & & & & & & & & & . & , & \\
\hline & . & & & & & & & & & & & & & . & . & \\
\hline & . & & & & & & & & & & & & . & . & & \\
\hline & . & & . & & & & & & & & & & . & . & . & \\
\hline
\end{tabular}

talia, and (4) the amphisteginids Amphistegina and Asterigerina. The coarse, graded, lower part of Core 129-802A-28R contains all four larger foraminiferal groups, in addition to a number of smaller benthic species. Upsection the abundance of the larger foraminifers decreases to zero with diminishing grain size to Section 129-802A-27R-3.

A number of paleoenvironments are represented by the graded bed and these are reflected by the larger foraminiferal facies. Lepidocyclina sumatrensis and Heterostegina borneensis are reported to be typical of sea grass communities from water depths of less than $12 \mathrm{~m}$ developed in normal oceanic salinities, but under sheltered conditions (Chaproniere, 1975). Living Heterostegina is most commonly found in the shallower parts of the euphotic zone $(15-30 \mathrm{~m})$, where it is associated with algal symbionts (Lutze etal., 1971). Rotalia, in contrast, is typical of high-energy reef environments today, where it is found in up to $40-\mathrm{m}$ water depth
(Murray, 1973). The amphisteginids are also fairly typical of reefs, but are also adapted to a range of other environments including beach, lagoon, and even outer slope and deeper water habitats (Todd, 1976). Amphisteginid test morphology may provide some general information about the paleoenvironment(Todd, 1976): inflated robust forms are more typical of the higherenergy (beach) environments whereas flattened morphotypes are characteristic of lagoonal and outer slope areas.

The larger foraminiferal assemblages in the graded bed at the top of Unit III clearly indicate the development of a reef and associated environments during the early Miocene. The grading of the single bed suggests it was deposited in a single episode, although it is not possible to determine the duration of the resedimentation episode. The absence of obviously younger planktonic microfossils in the graded bed suggests that redeposition was contemporaneous, or soon after 
Table 1 (continued).

\begin{tabular}{|c|c|c|c|c|c|c|c|c|c|c|c|}
\hline $\begin{array}{l}\text { Hole 802A } \\
\text { Core, section: } \\
\text { Interval }(\mathrm{cm}): \\
\text { No. of species: }\end{array}$ & $35 \mathrm{R}-\mathrm{CC}$ & $\begin{array}{c}36 \mathrm{R}-\mathrm{CC} \\
1\end{array}$ & $\begin{array}{c}37 \mathrm{R} \cdot \mathrm{CC} \\
0\end{array}$ & $\begin{array}{c}38 R-1 \\
70-73 \\
13\end{array}$ & $\begin{array}{c}38 \mathrm{R}-2 \\
48-52 \\
8\end{array}$ & $\begin{array}{c}38 \mathrm{R}-3 \\
101-105 \\
2\end{array}$ & $\begin{array}{c}38 R-4 \\
115-118 \\
19\end{array}$ & $\begin{array}{c}38 R-5 \\
54-59 \\
13\end{array}$ & $\begin{array}{c}38 \mathrm{R}-6 \\
5-8 \\
0\end{array}$ & $\begin{array}{c}39 \mathrm{R}-1 \\
49-51 \\
0\end{array}$ & $\begin{array}{c}39 R-1 \\
60-61 \\
1\end{array}$ \\
\hline \multicolumn{12}{|l|}{ "Larger" foraminifers } \\
\hline Amphistegina hauerina & & . & & & & & & & & & . \\
\hline Asterigerina marshallana & & & . & & & & & & & & . \\
\hline Asterigerina tentoria & & . & . & & . & & & & & & . \\
\hline Heterostegina borneensis & & . & & . & & & & & . & & . \\
\hline Lepidocyclina sumatrensis & & & & & & & & & & & \\
\hline Pararotalia armata & & & . & & & & & & . & & \\
\hline Rotalia tectoria & & . & . & & & & & & . & & . \\
\hline \multicolumn{12}{|l|}{ "Smaller" foraminifers } \\
\hline Angulogerina angulosa & & & & & & & i & . & . & & \\
\hline Aragonia oue:zaensis & & & & & & & $\mathbf{R}$ & $\mathrm{F}$ & . & & \\
\hline Aragonia velascoensis & & & & & & & $\mathrm{F}$ & $\mathbf{R}$ & & & \\
\hline Bolivina indet. spp. & . & & & & & & $\mathrm{F}$ & $\mathrm{F}$ & & & \\
\hline Bolivina sp. 1 & & & & & & & $\mathrm{~F}$ & $\mathrm{~F}$ & & & . \\
\hline Bulimina tuxpamensis & & & & & & & . & & & & \\
\hline Cassidulina subtumida & & . & . & & & & . & $\mathrm{F}$ & & & . \\
\hline Cibicides ribbingi & & & & & & . & $\mathrm{F}$ & & & & . \\
\hline Cibicides spp. & & . & & & & . & $\mathrm{F}$ & $\mathrm{F}$ & & & $\mathbf{P}$ \\
\hline Cibicidoides voltziana & & $\mathrm{P}$ & & & & & $\mathrm{F}$ & c & & & . \\
\hline Dentalina spp. & & & & & & & $\mathrm{R}$ & & & & \\
\hline Epistomina caracolla & & & & & & & $\mathrm{c}$ & $\mathrm{R}$ & & & . \\
\hline Gaudrvina pyramidata & & & & & & & $\mathrm{R}$ & $\mathrm{F}$ & . & & . \\
\hline Globorotalites micheliamus & & & & & & & $\ddot{R}$ & & & & \\
\hline Gyroidinoides beisseli & & & & & & . & $\mathrm{F}$ & & & & \\
\hline Gyroidinoides cf. praeglobosus & & & & & & & & & & & \\
\hline Lenticulina sp. I & & & & & & & & & & & . \\
\hline Lenticulina spp. & & & & & & & . & . & & & \\
\hline Loxostomum eleyi & & & & & & & $\mathrm{F}$ & $\mathrm{F}$ & & & . \\
\hline Oridorsalis umbonatus & & & & & & . & & & & & \\
\hline Orthomorphina aff. antillea & & . & & & & & $\mathrm{F}$ & & & & . \\
\hline Orthomorphina cf. columnaris & & $=$ & & & & & & & & & . \\
\hline Planulina cf. wuellerstorfi & & & & & & & $\mathrm{F}$ & & & & . \\
\hline Pleurostomella cf. alternans & & & & & & & $\mathrm{F}$ & & & & . \\
\hline Praebulimina cushmani & & & & & & & $\mathrm{F}$ & c & . & & \\
\hline Pullenia minuta & & & & & & & $\mathrm{R}$ & . & . & & \\
\hline Reussella cf. pseudospinulosa & & & & & & & & $\mathbf{R}$ & . & & \\
\hline Siphonina cf. temuicarinata & . & & & & & & & & . & & \\
\hline Stilostomella lepidula & & & & & & & . & & , & & \\
\hline Stitostomella pseudoscripta & & & & & & & $\mathrm{F}$ & $\mathrm{F}$ & & & \\
\hline Stilostomella sp. 1 & & & & & & & : & & & & \\
\hline \multicolumn{12}{|l|}{ Deep-water agglutinants } \\
\hline Dendrophrya latissima/excelsa & & & & $A$ & A & c & & & & & . \\
\hline Glomospira chamides & & & & $\hat{R}$ & & . & & & & & . \\
\hline Haplophragmoides biumbilicalis & & . & & $\mathrm{R}$ & . & & & & & & . \\
\hline Haplophragmoides herbichi & & & & $\mathrm{R}$ & $\mathrm{R}$ & & & . & & & \\
\hline Haplophragmoides spp. & & & & . & $\mathrm{F}$ & & & & & & \\
\hline Hormosina crassa & & . & & $\mathrm{F}$ & & & & & & & \\
\hline Hormosina ovulum & . & & & $\mathrm{F}$ & & & & & & & \\
\hline Hyperammina dilatata & . & & & $\mathrm{F}$ & & & & & & & \\
\hline Paratrochamminoides corpulentus & & & & $\mathrm{F}$ & $\mathrm{F}$ & & & & & & \\
\hline Paratrochamminoides intricatus & & & & $\mathrm{F}$ & & & & & & & . \\
\hline Plectorecurvoides rotundus & & & & $\mathrm{R}$ & $\mathrm{F}$ & & & & & & . \\
\hline Praecystammina globigerinaeformis & & & & $\mathbf{R}$ & : & $\therefore$ & & & . & . & \\
\hline Rhizammina sp. 1 & & & & C & c & $\mathrm{F}$ & & & & & \\
\hline Saccammina sphaerica & & & & $\mathrm{R}$ & . & & & & & & \\
\hline Subreophax scalaris & & & & & $\mathbf{R}$ & & & & & & . \\
\hline Trochammina depressa & & & & & R & & & & & & \\
\hline
\end{tabular}

the original deposition. The cause of the erosional event is not clear, since it apparently does not correlate with any of the erosional events documented for other sites in the western Pacific, although there still remains some question concerning the age of the Heterostegina-Lepidocyclina skeletal debris reported by Premoli Silva and Brusa (1981) from the Nauru Basin (Site 462).

A global sea-level highstand is a possible cause for the early Miocene erosion/redeposition event seen at Site 802. Although siliciclastic turbidites may result from progradation during sea-level lowstands, Austin et al. (1985) noted that the response of carbonate banks to changes in sea level was the opposite to that of clastic dominated slopes; at Site 632 (Little Bahama Bank) the maximum rates of turbidite deposition off carbonate bank margins corresponded to sealevel highstands. Haq et al. (1987) predicted short-term sea-level highstands for the earliest and latest early Miocene (early Aquitanian and middle Burdigalian); however, the biostratigraphic resolution in
Core 129-802A-28R is not detailed enough to enable detailed comparison with the published sea-level curves.

A second graded bed occurs in the upper part of Core 129-802A$27 \mathrm{R}$. The foraminiferal assemblage in this graded bed is identical to the one in the underlying core, indicating that a similar range of paleoenvironments existed. The graded bed in this core, however, is associated with volcaniclastic debris (glass and igneous rock fragments), which indicates that active volcanism was the probable trigger for the debris flow (Fig. 5), perhaps combined with sea-level highstand. The nearest likely source for the volcanism is the Caroline Islands, approximately $350 \mathrm{~km}$ distant from Site 802 .

\section{CONCLUSIONS}

The DWAF of Site 802 date the pelagic claystone of Unit IV as late Campanian and enable correlation to be made with sites in the Pigafetta 


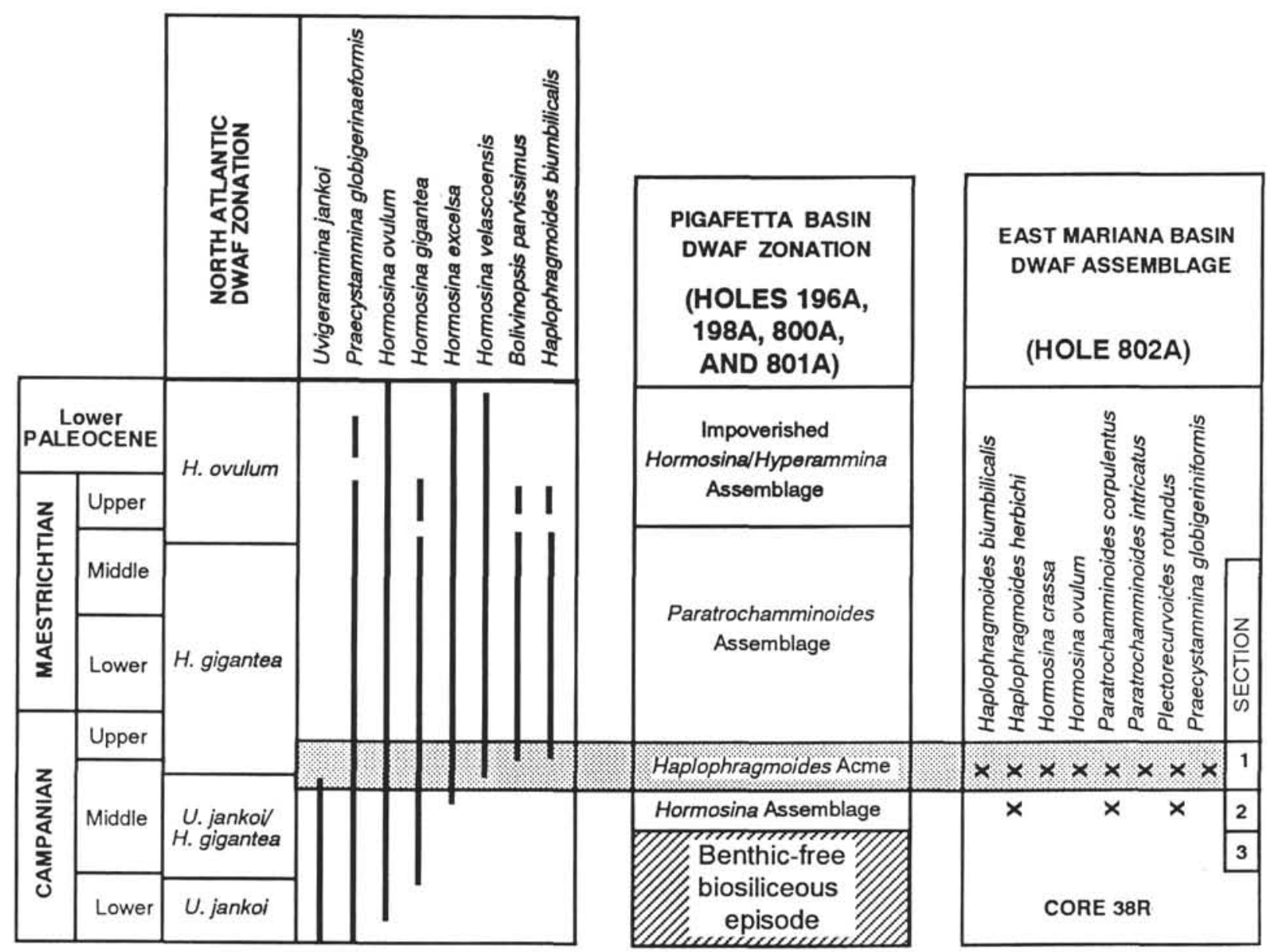

Figure 3. Correlation of deep-water agglutinated foraminiferal assemblages (DWAF) of Hole 802A with sites in the Pigafetta Basin and North Atlantic.

Basin and the North Atlantic. Late Campanian distal turbidites at Site 802 contain calcareous benthic foraminifers redeposited from bathyal to abyssal source areas. Assemblages of tubular abyssal agglutinated foraminifers indicate the final phases of distal turbidite sedimentation rather than a Late Cretaceous biosiliceous event. Autochthonous abyssal agglutinated assemblages were of moderate to low diversity during the late Campanian. Early Miocene erosion and redeposition of reefs and associated environments, dated by larger foraminifers, may correspond to a global sea-level highstand. Further erosion and redeposition at approximately the same time could have resulted from volcanic activity at or near the Caroline Islands.

\section{ACKNOWLEDGMENTS}

I thank the Ocean Drilling Program for inviting me to participate on Leg 129 aboard JOIDES Resolution. Frank Thomas (Geological Survey of Canada) is thanked for his help with sample preparation and the SEM photography. Wolfgang Kuhnt (Dalhousie University) gave useful help with the taxonomy of the deep-water agglutinated foraminifers and is also thanked for his assistance with the transmitted light photography. I would like to thank W. A. Berggren, F. M. Gradstein, and an anonymous reviewer for their comments on this manuscript. Financial support for this research was provided by an NSERC Collaborative Special Project Grant awarded to Felix Gradstein (Dalhousie University).

\section{TAXONOMIC NOTES}

Included in the alphabetic listing of species are the original and current name of the species. Full synonomy listing is not provided, although additional references relating to occurrence of certain stratigraphically important species in the Indo-Pacific region are given.

$$
\text { Amphistegina hauerina d'Orbigny, } 1846
$$

$$
\text { (Pl. 4, Figs. 13, 14) }
$$

Amphistegina hauerina d'Orbigny, 1846, p. 207, pl. 12, figs. 3-5.

Amphistegina hauerina d'Orbigny, Larsen, 1978, p. 224, pl. 2, figs. 1, 4.

Remarks. The test is smooth and biconvex with slightly raised glassy bosses on the spiral and umbilical sides. Fifteen chambers are in the final whorl, and raised sutures are sharply angled at one-half to two-thirds the distance from the umbilicus to the angled periphery. The spiral side has interseptae and supplementary chambers. The aperture is interiomarginal with a small lip. A small papillate area is developed in front of the aperture. Larsen (1978) emended the taxonomy of this species to include A. mamillata and A. rugosa. Specimens from Hole 802A resemble Larsen's (1978) figures closely, and are also quite similar to the recent form $A$. radiata. A. hauerina differs from A. bikiniensis, described from the Bikini Atoll drill hole, in its angled rather than curved sutures and reduced papillate area.

\section{Angulogerina angulosa (Williamson, 1858)}

$$
\text { (PI. 2, Figs. 15, 16) }
$$

Uvigerina angulosa Williamson, 1858, p. 69.

Angulogerina angulosa (Williamson, 1858), Loeblich and Tappan, 1985, p. 785 , pl. 574 , figs. $5-9$.

Remarks. Specimens are triangular in cross section, with each apex of the triangle formed with strong longitudonal ridges that extend the length of the test, up onto the apertural neck. Shorter costae ornament individual chambers. The apertural neck is surrounded by a thickened rim. In some specimens, the longitudinal ridges are paired. 
Aragonia ouezzanensis (Rey, 1955)

(Pl. 3, Fig. 7)

Bolivinoides ouezzanensis Rey, 1955, p. 210, pl. 12, fig. 2

Aragonia ouezzanensis (Rey), Sliter, 1977, p. 674, pl. 7, figs. 9, 10.

Aragonia ouezzanensis (Rey), Tjalsma and Lohmann, 1983, p.4, pl.5, figs. 4a-c.

Remarks. Distinguished from A. velascoensis by its greater apical angle, greater thickness, and shorter length. Van Morkhoven et al., (1986) regarded A. ouezzanensis and A. velascoensis as conspecific; however, no gradation is apparent between the two forms in material from Hole $802 \mathrm{~A}$, and they are treated as separate species.

Aragonia velascoensis (Cushman, 1925) (PI. 3, Fig. 6)

Textularia velascoensis Cushman, 1925, p. 18, pl. 3, fig. 1.

Aragonia velascoensis (Cushman, 1925), Tjalsma and Lohmann, 1983, p. 5 , pl. 4 , fig. 6 .

Aragonia velascoensis (Cushman, 1925), van Morkhoven et al., 1986, p. 340, pl. 111A, Figs. 1a, b, 2a, b, 3 .

Remarks. A. velascoensis was recognized by its broad apical angle and its raised limbate sutures. Western Pacific specimens are ornamented by raised projections extending from the sutures onto the surface of the earlier chambers.

\section{Asterigerina marshallana Todd and Post, 1954}

$$
\text { (Pl. 4, Fig. 3) }
$$

Asterigerina marshallana Todd and Post, 1954, p. 562, pl. 201, figs. 3a-c.

Remarks. A. marshallana is unequally biconvex, with about 12 chambers in the final whorl. Sutures are indistinct. Well-developed rows of papillae occur in front of the aperture. The Hole 802A specimens compare with Todd and Post's (1954) illustrated specimens from the Bikini Island drill hole.

\section{Asterigerina tentoria Todd and Post, 1954}

$$
\text { (Pl. 4, Figs. 4, } 8
$$

Asterigerina tentoria Todd and Post, 1954, p. 562, pl. 201, figs. 2a-c.

Remarks. This species has a strongly convex ventral side with a glassy umbilical boss, sutures flush, six to eight chambers in the final whorl, and stellate supplementary chambers extending to about halfway to the periphery. The periphery is sharply angled.

\section{Bolivina sp. 1}

(PI. 2, Fig. 8)

Remarks. The test has six to eight pairs of chambers, sutures curved and gently depressed, periphery slightly lobulate in later part of test, and the wall is smooth and finely perforate. Specimens are 400-500 $\mu \mathrm{m}$ long, 180-225 $\mu \mathrm{m}$ wide.

Bulimina tuxpamensis Cole, 1928

Bulimina tuxpamensis Cole, 1928, p. 212, pl. 32, fig. 23. Sliter, 1981, p. 343 , pl. 5 , figs. 2,3 .

Bulimina miolaevis Finlay, Boltovskoy, 1978, p. 150, pl. 1, figs. 40, 41

Bulimina tuxpamensis Cole, van Morkhoven et al., 1986, p. 155, pl. 5la, figs. $1-4$, pl. $51 \mathrm{~b}$, figs. 1a, b, 2.

Remarks. The test is stout, enlarged abruptly from the early chambers. The chambers are inflated, sutures slightly depressed, and wall finely perforate and lacking any ornament.

\section{Cassidulina subtumida Cushman, 1933}

$$
\text { (Pl. 3, Fig. 8) }
$$

Cassidulina subtumida Cushman, 1933, p. 93, pl. 10, fig. 5. Cassidulina subtumida Cushman, 1933, Boltovskoy, 1978, p. 155, pl. 3, fig. 1.

Remarks. The specimens are small, $300-350 \mu \mathrm{m}$ in diameter, with slightly depressed sutures and a smooth wall.
Cibicides ribbingi Brotzen, 1936

(Pl. 2, Figs. 19, 20)

Cibicides ribbingi Brotzen, Sliter 1986, p. 354, pl. 8, figs. 1-5.

Remarks. Specimens from Hole 802A are plano-convex, flat on the spiral side, with an angular periphery. The tests have a regular curved peripheral outline in early chambers, becoming irregular to lobulate in the later chambers of the final whorl. Considerable variability in the test outline was seen in the specimens from Hole $802 \mathrm{~A}$.

\section{Cibicides voltziana (d'Orbigny)}

(PI. 3, Figs. 1-3)

Rotalina voltziana d'Orbigny

Cibicides voltziana (d’Orbigny), Sliter, 1986, p. 344, pl. 8, figs. 6-9.

Remarks. The test is small and plano-convex, with 10-12 chambers in the final whorl. The sutures are strongly curved back, flush between early chambers, become depressed between later chambers, and are strongly incised between the penultimate and final chambers. The final chamber extends short of the umbilicus, which commonly has a prominent umbo. This species is distinguished from the morphologically similar Cibicidoides eocaenus by its smaller size.

\section{Dentalina spp}

(Pl. 2, Fig. 1)

Remarks. Incomplete specimens of Dentalina were found, but could not be identified to species level.

\section{Dendrophrya latissima/Dendrophrya excelsa fragments}

$$
\text { (Pl. 1, Figs. 2, 3) }
$$

Dendrophrya latissima Grzybowski, Kaminski et al., 1988, p. 182, pl. 1, fig. 6. Dendrophrya excelsa Grzybowski, 1898, p. 272, pl. 10, figs. 1-4.

Remarks. The agglutinated tubular tests show considerable morphologic variability with respect to grain size, tube width, degree of compression, and curvature of the tube. Two end-member morphotypes were recognized: (1) strongly compressed, coarsely agglutinated, and straight-assigned to $D$. latissima (Pl. 1, Fig. 2) and (2) weakly compressed, finely agglutinated, and curved-assigned to D. excelsa (Pl. 2, Fig. 3).

\section{Epistomina cf. caracolla (Roemer, 1841)}

$$
\text { (Pl. 4, Figs. 1, 2) }
$$

Gyroidina caracolla Roemer, 1841, p. 97, pl. 15, figs. 22a-c. Epistomina caracolla (Roemer, 1841), Ascoli, 1976, p. 746, pl. 3, fig. 10 Epistomina caracolla (Roemer, 1841), Hart, 1984, p. 293, pl. 1, figs. 13-19, pl. 2, fig. 15 .

Remarks. Epistomina cf. caracolla has a bi-convex test, a keeled periphery, and a conical ventral surface with a glassy umbilical boss. The wall is smooth on ventral and dorsal surfaces. Lateromarginal apertures were seen in last few chambers. Specimens from Hole $802 \mathrm{~A}$ are abraded and show clearly the internal partition extending from the peripheral margin and across the wall of the previous chamber. They differ from $E$. caracolla in that the primary aperture appears as an elongate slit, divided in two by the internal partition; this feature may be related to dissolution.

\section{Gaudryina pyramidata Cushman, 1926}

$$
\text { (Pl. 1, Fig. 19) }
$$

Gaudryina laevigata Franke var. pyramidata Cushman, 1926, p. 587, pl. 16, figs. $8 \mathrm{a}, \mathrm{b}$.

Gaudryina pyramidata Cushman, 1926, Sliter, 1977, p. 675 , pl. 2, fig. 9.

Gaudryina pyramidata Cushman, 1926, Hemleben and Troester, 1984, p. 518 , pl. 4, figs. 16, 17.

Gaudryina pyramidata Cushman, 1926, Kaminski et al., 1988, p. 194, pl. 8, fig. 7 .

Remarks. The triserial test becomes biserial, with sharply angled edges. The aperture is interiomarginal. The test is agglutinated from fine sand grains.

Globorotalites michelianus (d'Orbigny)

(Pl. 3, Figs. 14, 19) 
Globorotalites michelianus (d'Orbigny), Sliter, 1986, p. 345, pl. 11, figs. 6-8.

Remarks. The single specimen recovered from Hole $802 \mathrm{~A}$ is identified by its conical shaped plano-convex test and its particularly high spire.

Glomospira charoides (Jones and Parker, 1860)

$$
\text { (Pl. 1, Fig. 6) }
$$

Trochammina squamata Jones and Parker var. charoides Jones and Parker, 1860 , p. 304.

Glomospira charoides (Jones and Parker), Kaminski et al., 1988, p. 185, pl. 3 , figs. 14,15 .

Glomospira charoides (Jones and Parker), Moullade et al., 1988, p. 363, pl. 1 , fig. 6 .

Remarks. The test is finely agglutinated and well preserved.

$$
\text { Gyroidinoides beisseli (Schijfsma) }
$$$$
\text { (Pl. 3, Figs. 15, 16) }
$$

Eponides beisseli Schijfsma

Gyroidinoides beisseli (Schijfsma), Sliter, 1977, p. 675, pl. 10, figs. 3-6.

Gyroidinoides beisseli (Schijfsma), Sliter, 1984, p. 345, pl. 11, figs. 17-18.

Remarks. The biconvex test has six to seven chambers in the final whorl. Sutures are indistinct, but slightly depressed at the periphery. The aperture extends from the umbilicus to the periphery, and is bordered by a lip over most of its length.

\section{Gyroidinoides cf. praeglobosus (Brotzen)}

$$
\text { (PI. 3, Figs. 17, 18) }
$$

Gyroidina praeglobosus Brotzen

Gyroidinoides praeglobosus (Brotzen), Sliter, 1977, p. 675, pl. 11, figs. 3, 6 .

Remarks. The inflated biconvex test has flush sutures and an aperture with a bordering lip. The specimen recovered from Hole $802 \mathrm{~A}$ is large for the species, with a diameter of $700 \mu \mathrm{m}$

Haplophragmoides biumbilicalis Krasheninnikov, 1973

$$
\text { (PI. 1, Figs. 10, 11) }
$$

Haplophragmoides biumbilicalis Krasheninnikov, 1973, p. 208, pl. 1, figs. $3 \mathrm{a}-\mathrm{b}, 4$.

Remarks. Five inflated chambers are in the final whorl, with a deep umbilicus. Specimens from Site 802 are small (maximum diameter $200 \mu \mathrm{m}$ ), and compare well with those illustrated in Krasheninnikov (1973).

Haplophragmoides herbichi Neagu, 1968

(PI. 1, Fig. 12)

Haplophragmoides herbichi Neagu, 1968, P. 238, pl. 1, figs. 9-12.

Haplophragmoides $\mathrm{cf}$. herbichi Neagu, Moullade et al., 1988, p. 365, pl. 6 , figs. 1, 2.

Remarks. The test is planispirally coiled with eight to nine chambers in the final whorl. Sutures are flush, resulting in a rounded periphery. The umbilicus is deeply excavated.

\section{Heterostegina borneensis Van der Vlerk, 1929}

(Pl. 4, Fig. 10; Pl. 5, Figs. 6, 7)

Heterostegina borneensis Van der Vlerk, 1929, p. 16, figs. 6a-c, 25a-b.

Heterostegina borneensis Van der Vlerk, Cole, 1957, p. 757-759, pl. 237. figs. 1-23.

Remarks. The Hole 802A specimens have an inflated lenticular central part and a narrow, flattened rim. Tests are smooth and lack papillae. The embryonic chambers consist of a protoconch with partly enveloping deuteroconch.

\section{Hormosina crassa Geroch, 1966}

(Pl. 1, Fig. 7)

Hormosina ovulum crassa Geroch, 1966, p. 438, figs. 6(19,21-26), 7(21-23). Hormosina crassa Geroch, Moullade et al., 1988, p. 365, pl. 2, figs. 4-6. Hormosina crassa Geroch, Kuhnt, 1990, pl. 2, fig. 5.
Remarks. The ovoid to spherical test is distinguished from other species of Hormosina by its more coarsely agglutinated wall.

Hormosina ovulum (Grzybowski, 1898)

$$
\text { (Pl. 1, Fig. 8) }
$$

Reophax ovulum Grzybowski, 1896, p. 276, pl. 8, figs. 19-21.

Hormosina ovulum ovulum (Grzybowski), Kuhnt and Kaminski, 1990, p. $474-475$, pl. 1, figs. c-e.

Remarks. Specimens from Site 802 have slightly drawn out chambers, relatively broad chamber connections, and may be transitional to $H$. ovuloides.

\section{Hyperammina dilatata Grzybowski, 1896}

(Pl. 1, Fig. 4)

Hyperammina dilatata Grzybowski, 1896, pp. 474-475, pl. 8, fig. 17.

Hyperammina dilatata Grzybowski, 1896, Hemleben and Troester, 1984, p. 520 , pl. 1, figs. $7-9$.

Hyperammina dilatata Grzybowski, 1896, Kaminski et al., 1988, p. 184, pl. 2, figs. 1-2.

Remarks. The small, smooth-walled tubes with regular chamber swellings are separated by slight constrictions. Fragments from Hole 802A contain up to three chambers. Specimens are similar to Hormosina excelsa; however, they do not possess the "pear"-shaped chambers typical of $H$. excelsa. The broad apertural openings distinguish Hyperammina dilatata from the otherwise similar Hormosina ovuloides.

Lenticulina sp. 1

(Pl. 2, Figs. 2, 3)

Description. The swollen test has a thickness three-quarters the test diameter, nine chambers in the final whorl, glassy umbones, and a bluntly angled periphery. The wall is smooth with the exception of the elevated, limbate, and gently curved sutures.

Remarks. Distinguished by its inflated test.

$$
\text { Lepidocyclina (Nephrolepidina) sumatrensis (Brady, 1875) }
$$$$
\text { (Pl. 4, Figs. 12, 15-17; Pl. 5, Figs. 1-5) }
$$

Orbitoides sumatrensis Brady, 1875, p. 536, pl. 24, figs. 3a-b.

Lepidocyclina sumatrensis (Brady), Caudri, 1939, pp. 185-197, 243, 247, 250, 251 , pl. 7, figs. $36-41,44$.

Lepidocyclina parva Oppenoorth, 1918, p. 255, pl. 8, figs. 11-12; pl. 9, fig. 9. Lepidocyclina sumatrensis (Brady), Cole, 1957, pp. 773-775, pl. 239, figs.

$1-4$; pl. 241, figs. $1-30$; pl. 242 , figs. $3-20$.

Remarks. The test is distinguished by the nephrolepidine embryonic chambers and short arcuate to diamond-shaped equatorial chambers. Thin sections of some specimens feature four well-developed periembryonic chambers - a primary auxiliary chamber on each side of the dividing wall of the embryonic chambers and two adauxiliary chambers adjoined to the dueteroconch (Pl. 5, Fig. 1). Other specimens do not have such well-developed periembryonic chambers (PI. 5, Figs. 2 and 3). Hole 802A specimens are $2.0-4.0 \mathrm{~mm}$ in diameter and show considerable variability in the development of pillars, seen both internally and externally. Cole (1957) placed $L$. parva in synonomy with $L$. sumatrensis, and the same strategy has been adopted here to account for the variability seen in specimens examined in thin section. There is not sufficient material available to study the taxonomic relations of Hole 802A specimens in further detail.

$$
\text { Loxostomum eleyi (Cushman, 1927) }
$$$$
\text { (Pl. 3, Fig. 4) }
$$

Bolivinita eleyi Cushman, 1927, p. 490.

Loxostomum eleyi (Cushman), Sliter, 1986, p. 355, pl. 9, fig. 12

Loxostomum eleyi (Cushman), Sliter, 1977, p. 675, pl. 7, fig. 8.

Remarks. Loxostomum eleyi is quadrate in section, with carinate peripheral margins. The chambers are strongly arched, with limbate, but flush, sutures.

Oridorsalis umbonatus (Reuss, 1851)

(Pl. 3, Figs. 11-13) 
Rotalina umbonata Reuss, 1851, p. 75, pl. 5, fig. 35.

Oridorsalis umbonatus (Reuss), Boltovskoy, 1977, p. 162, pl. 5, figs. 5, 6.

Oridorsalis umbonatus (Reuss), Douglas, 1973, p. 647 , pl. 13, figs. I-6, pl. 24 , figs. $11,12$.

Remarks. Specimens from Hole $802 \mathrm{~A}$ are well preserved and display the additional openings at the junction of the spiral and radial sutures on the spiral side, and the secondary aperture at the umbilicus to the rear of the final chamber.

\section{Orthomorphina cf, columnaris (Franke, 1936)}

$$
\text { (Pl. 2, Fig. 5) }
$$

Nodosaria columnaris Franke, 1936, p. 48, pl. 4, fig. 19.

Orthomorphina cf. columnaris (Franke), Boltovskoy, 1984, p. 163, pl. 5. fig. 18.

Remarks. This is a four-chambered form with only slight constrictions between the chambers. Eight to 10 longitudonal costae extend the full length of the test, up to the aperture. The wall is otherwise smooth. The aperture is round, surrounded by a remnant of a neck. The specimen recovered from Hole $802 \mathrm{~A}$ is also quite similar to O. modesta (Bermudez), but is larger, does not taper, and has fewer costae. It also resembles Nodosaria latejugata Gumbel, but does not possess the radiate aperture.

\section{Orthomorphina aff. antillea (Cushman, 1923)}

$$
\text { (Pl. 2, Fig. 4) }
$$

Nodosaria antillea Cushman, 1923, p. 91, pl. 14, fig. 9.

Orthomorphina antillea (Cushman), Boltovskoy, 1978, p. 162, pl. 5, figs. 7-11.

Remarks. Specimens from Hole $802 \mathrm{~A}$ are similar to $O$. antillea, but have less elongate chambers that are not so angled near their bases. The aperture lacks a tooth plate, distinguishing it from Stilostomella.

\section{Pararotalia armata (d'Orbigny)}

$$
\text { (PI. 4, Figs. 9, 11) }
$$

Rotalia armata d'Orbigny, 1826, p. 273, pl. 8, fig. 1.

Pararotalia armata (d'Orbigny), Loeblich and Tappan, 1957, p. 9, pl. 5, fig. 2. Pararotalia armata (d'Orbigny), Le Calvez, 1949, p. 161, pl. 39, fig. 1.

Remarks. Stellate in outline, the test has eight to nine chambers in the final whorl, a sharply angled periphery, and a central peripheral spine on each chamber. The sutures are deep, and the umbilicus is filled with a large calcite boss. Specimens from Hole $802 \mathrm{~A}$ are commonly abraded and the spines are much reduced.

\section{Paratrochamminoides corpulentus Krasheninnikov, 1973}

$$
\text { (Pl. 1, Figs. 13, 14) }
$$

Paratrochamminoides corpulentus Krasheninnikov, 1973, p. 212, pl. 3, figs. $3 \mathrm{a}, \mathrm{b}, \mathrm{c}$.

Paratrochamminoides semipellucidus Krasheninnikov. Hemleben and Troster, 1984 , p. 520 , pl. 3 , figs. $12-14$.

Remarks. Oval in outline with elongate chambers, it lacks an excavated umbilicus. The wall is smooth and finely agglutinated.

\section{Paratrochamminoides intricatus Krasheninnikov, 1973}

$$
\text { (P1. 1, Fig. 15) }
$$

Paratrochamminoides intricatus Krasheninnikov, 1973, p. 212, pl. 3, figs. $2 \mathrm{a}-\mathrm{c}$.

Paratrochamminoides intricatus Krasheninnikov, Hemleben and Troester, 1984 , p. 520 , pl. 3 , figs. $10,11$.

Remarks. The irregularly coiled globular chambers increase in size slowly as added. Specimens from Site 802 are larger than those previously reported from the Pacific or Indian Ocean, with a maximum diameter of $610 \mu \mathrm{m}$.

\section{Planulina cf. wuellerstorfi (Schwager)}

(P1. 2, Fig. 18)

Anomalina wuellerstorfi Schwager, 1866, p. 258, pl. 7, figs. 105, 107.

Planulina wuellerstorfi (Schwager), Douglas, 1973, p. 656, pl. 18, figs. 7-9. Planulina wuellerstorfi (Schwager), van Morkhoven et al., 1986, p. 49, pl. 14 figs. 1a, b, c, 2a, b, c.
Remarks. The spiral side is flat, umbilical side slightly convex, peripheral outline curved, and slightly lobulate in last few chambers. Ten chambers are in the final whorl, with sutures strongly curved and limbate on the spiral side. The umbilical side is smooth, whereas the spiral side is marked by coarse perforations. Specimens from Hole $802 \mathrm{~A}$ are small, with a diameter of up to $300 \mu \mathrm{m}$, but are otherwise identical to $P$. wuellerstorfi.

\section{Plectorecurvoides rotundus Krasheninnikov, 1974}

$$
\text { (Pl. 1, Fig. 16) }
$$

Plectorecurvoides rotundus Krasheninnikov, 1974, p. 641, pl. 5, figs. 4a, b, c, $5 \mathrm{c}, 6 \mathrm{c}$.

Remarks. Specimens from Hole $802 \mathrm{~A}$ are identical to those described from the Indian Ocean by Krasheninnikov (1974). The species has not previously been reported from the western Pacific. It is recognized by its inflated test and indistinct sutures.

\section{Pleurostomella cf. alternans Schwager}

(P1. 3, Fig. 5)

Pleurostomella alternans Schwager, Todd, 1957, pl. 67, figs. 12a, b, 13a, b. Pleurostomella bellarsdii Hantken, Berrgren, and Aubert, 1976, p. 338, pl. 1, fig. 7 .

Remarks. Small, with indistinct sutures and slightly lobulate periphery, the specimens from Hole $802 \mathrm{~A}$ compare well with $P$. alternans, but are considerably smaller (maximum length $350 \mu \mathrm{m}$ ).

\section{Praebulimina cushmani (Sandidge, 1932)}

$$
\text { (PI. 2, Figs. 6, 7) }
$$

Buliminella cushmani Sandidge, 1932, p. 280, pl. 42, figs. 18, 19. Praebulimina reussi (Morrow), Hemleben and Troester, p. 521, pl. 5, fig. 9. Praebulimina cushmani (Sandidge, 1932), Sliter, 1986, p. 343, pl. 4, figs. 18, 19.

Remarks. It is recognized by the four inflated chambers in the final whorl and the fine reticulate ornament on the chambers of the earlier whorls.

\section{Praecystammina globigerinaeformis Krasheninnikov, 1973}

$$
\text { (Pl. 1, Fig. 17) }
$$

Praecystammina globigerinaeformis Krasheninnikov, 1973, p. 211, pl. 3, figs. la, b, c, 2 .

Praecystammina globigerinaeformis Krasheninnikov, Hemleben, and Troester, 1984 , p. 521 , pl. 4 , fig. 8.

Praecystammina globigerinaeformis Krasheninnikov, Moullade et al., 1988, p. 366 , pl. 9 , figs. 9,10 .

Remarks. This species is represented by a single damaged specimen at Site 802 .

\section{Pullenia minuta Cushman}

$$
\text { (Pl. 3, Figs. 9, 10) }
$$

Pullenia minuta Cushman, Sliter, 1977, p. 690, pl. 8, figs. 8, 9.

Pullenia minuta Cushman, Sliter, 1986, p. 345, pl. 9, fig. 10.

Remarks. The test has five chambers in the final whorl, with the aperture bordered by a lip. The sutures are slightly depressed; final chamber is as broad as it is high in apertural view. It is distinguished from $P$. quinqueloba by its smaller size.

$$
\text { Reussella cf. pseudospinulosa Troelsen, } 1937
$$

$$
\text { (Pl. 2, Fig. 14) }
$$

Reussella pseudospinulosa Troelsen, 1937, p. 260, (nomen nudum). Reussella pseudospinulosa Troelsen, Brotzen, 1945, p. 46, pl. 1, fig. 6.

Reussella cf. pseudospinulosa Troelsen, Hemleben, and Troester, 1984, p. 521, pl. 5 , fig. 18.

Remarks. The form seen at Hole $802 \mathrm{~A}$ is rather small for the species (207 $\mu \mathrm{m}$ in height). The edges have well-developed spines.

Rhizammina sp. 1

(Pl. 1, Fig. 1) 
Remarks. Thin-walled agglutinated tubes with circular cross sections are grouped together as Rhizammina spp. These are generally narrower than the tubes assigned to Dendrophrya, and have a test diameter of 100-130 $\mu \mathrm{m}$.

Rotalia tectoria Todd and Post, 1954

$$
\text { (Pl. 4, Figs. 5-7) }
$$

Rotalia tectoria Todd and Post, 1954, p. 561, pl. 202, figs. 2-4.

Remarks. The specimens are large, $0.9-1.5 \mathrm{~mm}$ in diameter. The arcuate peripheral outline becomes lobulate in final chambers of larger specimens, and is rounded to bluntly angled in axial view. Ten to 13 chambers are in the final whorl, increasing in size slowly as added. Dorsal sutures are indistinct between the early chambers, and become slightly incised between later chambers of large specimens. The ventral side has a large irregular umbilical plug containing three or more pustules. The wall of the test is ornamented with smaller pustules; Aperture not seen. Hole 802A specimens correspond well with those illustrated in Todd and Post (1954).

\section{Saccammina sphaerica Sars, 1872}

$$
\text { (Pl. 1, Fig. 5) }
$$

Saccammina sphaerica Sars, 1872, p. 250.

Saccammina sphaerica Sars, 1872, Hemleben and Troester, 1984, p. 522, pl. 1, fig. 15.

Saccammina sphaerica Sars, 1872, Moullade et al., 1988, p. 366, pl. 2, fig. 12.

Remarks. The test is spherical with a single aperture. It is distinguished from the morphologically similar species Hormosina gigantea by its smaller size ( $350 \mu \mathrm{m}$ diameter) and coarser wall.

\section{Siphonina cf. tenuicarinata Cushman, 1927}

(Pl. 2, Fig. 17)

Siphonina tenuicarinata Cushman, 1927, p. 166, pl. 26, figs. 11, 12. Siphonina cf. tenuicarinata Cushman, 1927, van Morkhoven et al., 1986, p. 206, pl. 70, figs. 1a, b, c, 2, 3, 4 .

Remarks. A single broken specimen was recovered from Hole 802A. It is coarsely perforate. The apertural neck and the keel are broken away, which makes precise identification difficult.

\section{Stilostomella lepidula (Schwager, 1866) (Pl. 2, Fig. 11)}

Nodosaria lepidula Schwager, 1866, p. 210, pl. 5, fig. 27, 28.

Stilostomella ex. gr. lepidula (Schwager), Boltovskoy, 1984, p. 170, pl. 7, figs. $32-46$.

Remarks. It is recognized by the well-developed spines arranged in two rows in the lower portion of the chambers; however, spines are absent from the initial chamber. The aperture possesses a tooth, which distinguishes it from the otherwise similar genus Orthomorphina.

$$
\text { Stilostomella pseudoscripta (Cushman, 1943) }
$$

(PI. 2, Figs. 9, 10)

Ellipsonodosaria pseudoscripta Cushman, 1943

Stilostomella pseudoscripta (Cushman), Sliter, 1986, p. 343, pl. 6, figs. 8, 9.

Remarks. It is recognized by its finely spinose surface ornament, and differs from S. subspinosa by its smaller size. Variation is noted in the degree of constriction between the chambers.

\section{Stilostomella sp. 1}

(Pl. 2, Fig. 12)

Remarks. The test is straight to slightly arcuate with six to seven globular chambers gradually increasing in size as added. The wall is smooth and the terminal aperture is rounded, with a tooth.

\section{Subreophax scalaris (Grzybowski, 1896)}

$$
\text { (PI. 1, Fig. 9) }
$$

Reophax guttifera Brady var. scalaris Grzybowski, 1896, p. 277, pl. 8, fig. 26. Reophax scalaris Gryzbowski, Hemleben, and Troester, 1984, p. 521, pl. 2, figs. 10,11 .

Subeophax scalaris Grzybowski, Kaminski et al. (in press), p. 187, pl. 2, figs. 16, 17 .

Remarks. The test is gently curved, with globular chambers.

Trochammina depressa Lozo, 1944

$$
\text { (PI. 1, Fig. 18) }
$$

Trochammina depressa Lozo, 1944, p. 552, pl. 2, figs. 4a, 5 , Trochammina depressa Lozo, Crittenden, 1981, p. 21, pl. 2, fig. 13. Trochammina depressa Lozo, Wightman, 1990, p. 757, pl. 1, fig. 14.

Remarks. The strongly compressed test has about six chambers in the final whorl and a lobulate periphery. The wall is coarsely agglutinated and rough in appearance.

\section{REFERENCES}

Adams, C. G., 1970. A reconsideration of the East Indian letter classification of the Tertiary. Bull. Brit. Mus. (Nat. Hist.), Geol., 19:87.

Austin, J. A., Schlager, W., Palmer, A. A., Comet, P. A., Droxler, A., Eberli, G., Fourcade, E., Freeman-Lynde, R., Fulthorpe, C. S., Harwood, G., Kuhn, G., Lavoie, D., Leckie, R. M., Melillio, A. J., Moore, A., Mullins, H. T., Ravenne, C., Sager, W. W., Stewart, P., Verbeek, J. W., Watkins, D. K., and Williams, C., 1985. Megabanks found? flanks record sea level (ODP Leg 101, Sites 627, 628, 630, 631, 632, 633), Bahamas (Little Bahama Banks). Geotimes, 30:12-15.

Boltovskoy, E., 1978. Late Cenozoic benthonic foraminifera of the Ninetyeast Ridge (Indian Ocean). Mar. Geol., 26:139-175.

Cole, W. S., 1957. Larger foraminifera from Eniwetok atoll drill holes, Bikini and nearby atolls, Marshall Islands. Geol. Surv. Prof. Pap. U.S., 260V:742-783.

Coleman, P. J., 1963. Tertiary larger foraminifera of the British Solomon Islands, Southern Pacific. Micropaleontology, 9:1-38.

Chaproniere, G.G.H., 1975. Paleoecology of Oligo-Miocene larger foraminiferida, Australia. Alcheringa, 1:37-58.

1984. Oligocene and Miocene larger Foraminiferida from Australia and New Zealand. Bull.-Bur. Miner. Resour., Geol. Geophys. (Aust.), 188:1-98.

Haq, B. U., Hardenbol, J., and Vail, P. R., 1987. Chronology of fluctuating sea levels since the Triassic. Science, 235:1156-1167.

Kaminski, M. A., Grassle, J. F., and Whitlach, R. B., 1988. Life history and recolonization among agglutinated foraminifera in the Panama Basin. $A b h$. Geol. Bundesanst. Austria, 41:229-243.

Krasheninnikov, V. A., 1974. Upper Cretaceous benthonic agglutinated foraminifera, Leg 27 of the Deep Sea Drilling Project. In Veevers, J. J., Heirtzler, J. R., et al., Init. Repts. DSDP, 27: Washington (U.S. Govt. Printing Office), 631-662.

Kuhnt, W., 1990. Agglutinated foraminifera of the Western Mediterranean Upper Cretaceous pelagic limestone (Umbrian Apennines, Italy, and Betic Cordillera, Southern Spain). Micropaleontology, 36:297-330.

Kuhnt, W., Kaminski, M. A., and Moullade, M., 1989. Late Cretaceous deep-water foraminiferal assemblages from the North Atlantic and its marginal seas. Geol. Rundsch., 78:1121-1140.

Larsen, A., 1978. Phylogenetic and paleobiogeographical trends in the foraminiferal genus Amphistegina. Rev. Esp. Micropaleontol., 10:217-243.

Lutze, G. F., Grabert, B., and Siebold, E., 1971. Lebendbeobachtungen an Gross-Foraminiferen (Heterostegina) aus dem Persischen Golf. "Meteor" Forsch. Ergebn., C6:21-40.

Moullade, M., Kuhnt, W., and Thurow, J., 1988. Agglutinated benthic foraminifers from Upper Cretaceous variegated shales of the North Atlantic Ocean (DSDP Leg 93 and ODP Leg 103). In Boillot, G., Winterer, E. L., et al., Proc. ODP, Sci. Results, 103: College Station, TX (Ocean Drilling Program), 349-377.

Murray, J. W., 1973. Distribution and Ecology of Living Benthic Foraminiferids: London (Heinemann).

Premoli Silva, I., and Brusa, C., 1981. Shallow water skeletal debris and larger foraminifers from Deep Sea Drilling Project Site 462, Nauru Basin, Western Equatorial Pacific. In Larson, R. L., Schlanger, S. O., et al., Init. Repts. DSDP, 61: Washington (U.S. Govt. Printing Office), 439-473.

Shipboard Scientific Party, 1990. Site 802. In Lancelot, Y., Larson, R. L., et al., Proc. ODP, Init. Repts., 129: College Station, TX (Ocean Drilling Program), 171-243. 
Sliter, W. V., 1977. Cretaceous benthic foraminifers from the western South Atlantic Leg 39, Deep Sea Drilling Project. In Perch-Nielsen, K., Supko, P. R., et al., Init. Repts. DSDP, 39: Washington (U.S. Govt. Printing Office), 657-697.

1986. Cretaceous redeposited foraminifers from Deep Sea Drilling Project Site 585 in the East Mariana Basin, western Equatorial Pacific, and implications for the geologic history of the region. In Moberly, R., Schlanger, S. O., et al., Init. Repts. DSDP, 89: Washington (U.S. Govt. Printing Office), $327-361$.

Tjalsma, R. C., and Lohmann, G. P., 1983. Paleocene-Eocene bathyal and abyssal benthic foraminifera from the Atlantic Ocean. Micropaleontol. Spec. Publ., 4:1-90.

Todd, R., 1976. Some observations about Amphistegina (foraminifera). In Takayanagi, Y., and Saito, T. (Eds.), Progress in Micropaleontology: Selected Papers in Honour of Prof. Kiyoshi Asano. Spec. Publ. Am. Mus. Nat. Hist. N.Y., 382-394.

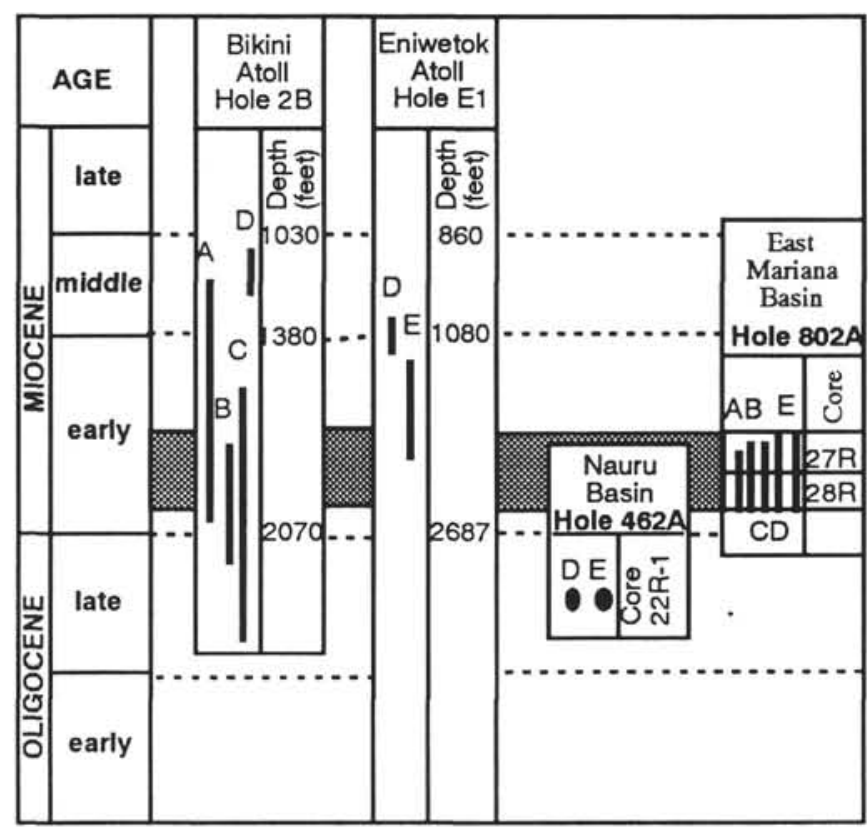

Figure 4. Correlation of larger foraminifer assemblages of Hole 802A with Bikini Atoll and Eniwetok Atoll drill holes. Key to species: $\mathrm{A}=$ Asterigerina tentoria $; \mathrm{B}=$ Asterigerina marshallana $; \mathrm{C}=$ Rotalia tectoria $; \mathrm{D}=$ Lepidocyclina sumatrensis $; \mathrm{E}=$ Heterostegina borneensis.
Todd, R., and Post, R. J., 1954. Smaller foraminifera from Bikini drill holes. In Bikini and Nearby Atolls: Part 4, Paleontology. Geol. Surv. Prof. Pap. U.S., 260-N.

Van der Vlerk, I. M., 1955. Correlation of the Tertiary of the Far East and Europe. Micropaleontology, 1:72-75.

van Morkhoven, FP.C.M., Berggren, W., and Edwards, A. S., 1986. Cenozoic cosmopolitan deep-water benthic foraminifera. Bull. Cent. Rech. Explor.Prod. Elf Aquitaine, 11.

Date of initial receipt: 3 June 1991 Date of acceptance: 17 March 1992 Ms 129B-123

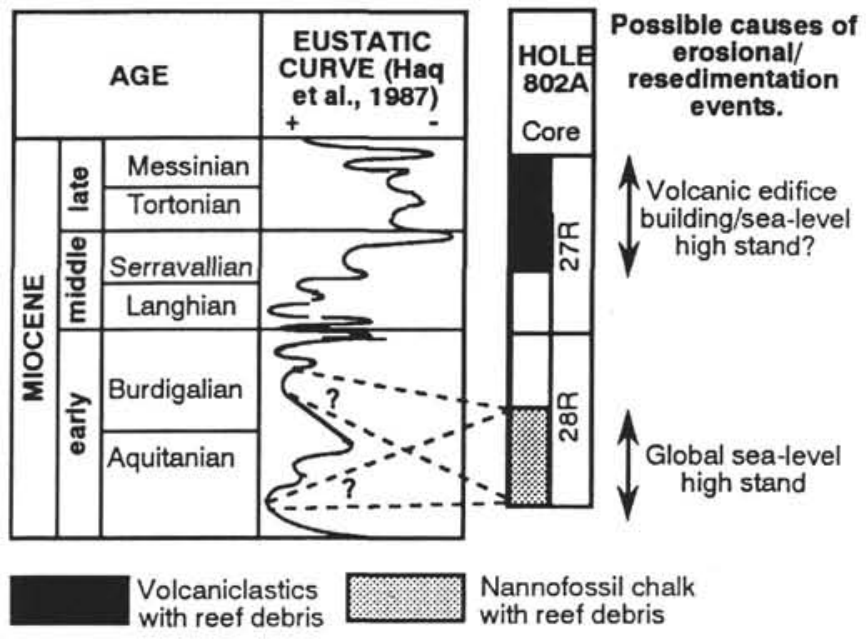

Figure 5. Possible causes of the early Miocene erosional/resedimentation events seen in Hole 802A. 


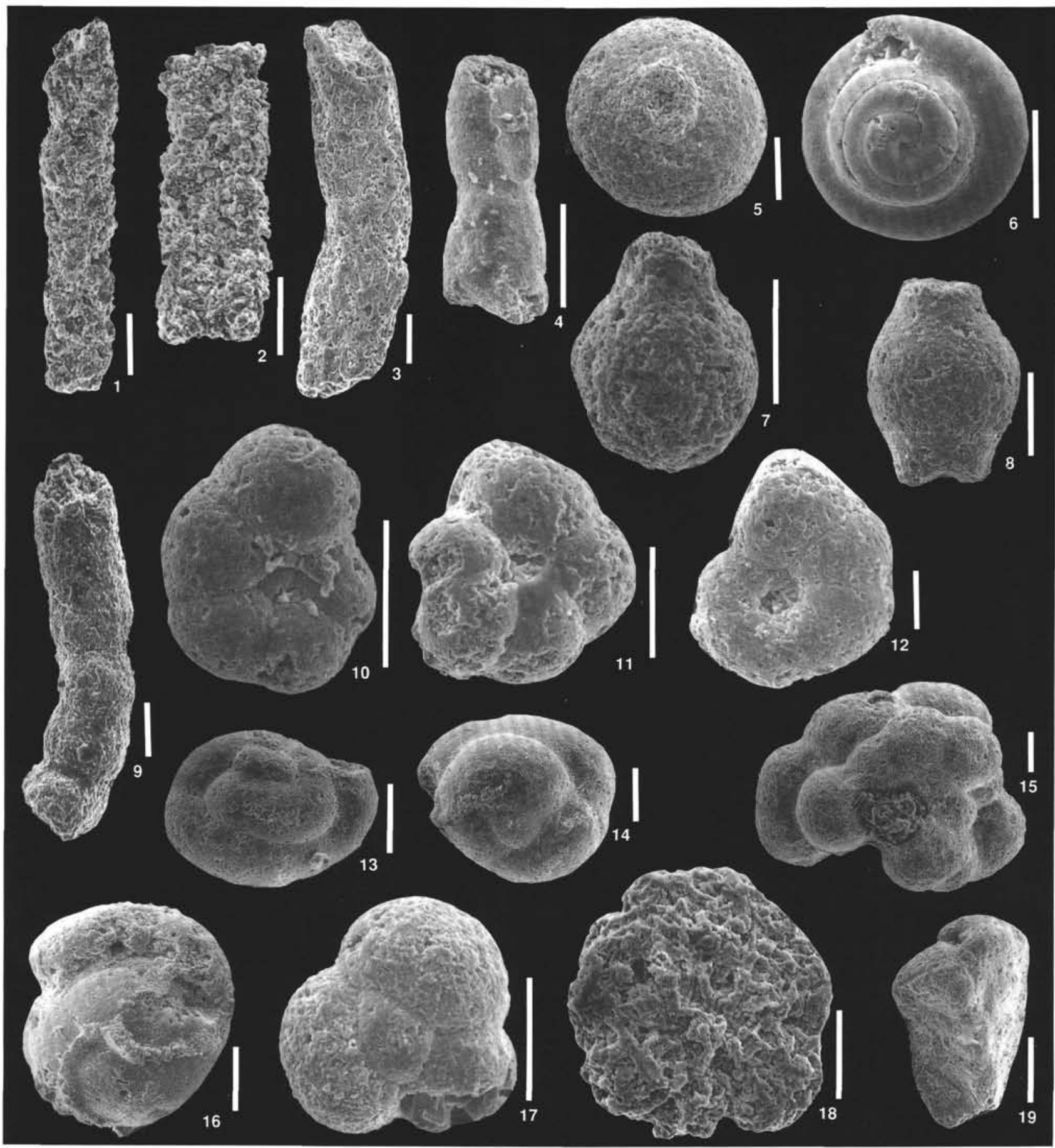

Plate 1. Scale bars $=100 \mu \mathrm{m}$. 1. Rhizammina sp., Sample 129-802A-38R-1, 70-73 cm. 2. Dendrophrya latissima, Sample 129-802A-38R-1, 70-73 cm. 3. Dendrophrya excelsa, Sample 129-802A-38R-1, 70-73 cm. 4. Hyperammina dilatata, Sample 129-802A-38R-1, 70-73 cm. 5. Saccammina spherica, Sample 129-802A-38R-1, 70-73 cm. 6. Glomospira charoides, Sample 129-802A-38R-1, 70-73 cm. 7. Hormosina crassa, Sample 129-802A-38R-1, 70-73 cm. 8. Hormosina ovulum, Sample 129-802A-38R-1, 70-73 cm. 9. Subreophax scalaris, Sample 129-802A-38R-2, 48-52 cm. 10,11. Haplophragmoides biumbilicalis, Sample 129-802A-38R-1, 70-73 cm. 12. Haplophragmoides herbichi, Sample 129-802A-38R-1, 70-73 cm. 13, 14. Paratrochamminoides corpulentus, Sample 129-802A-38R-1, 70-73 cm. 15. Paratrochamminoides intricatus, Sample 129-802A-38R-1, 70-73 cm. 16. Plectorecurvoides rotundus, Sample 129-802A-38R-2, 48-52 cm. 17. Praecystammina globigeriniformis, Sample 129-802A-38R-1, 70-73 cm. 18. Trochammina depressa, Sample 129-802A-38R-2, 48-52 cm. 19. Gaudryina pyramidata, Sample 129-802A-38R-5, 54-59 cm. 


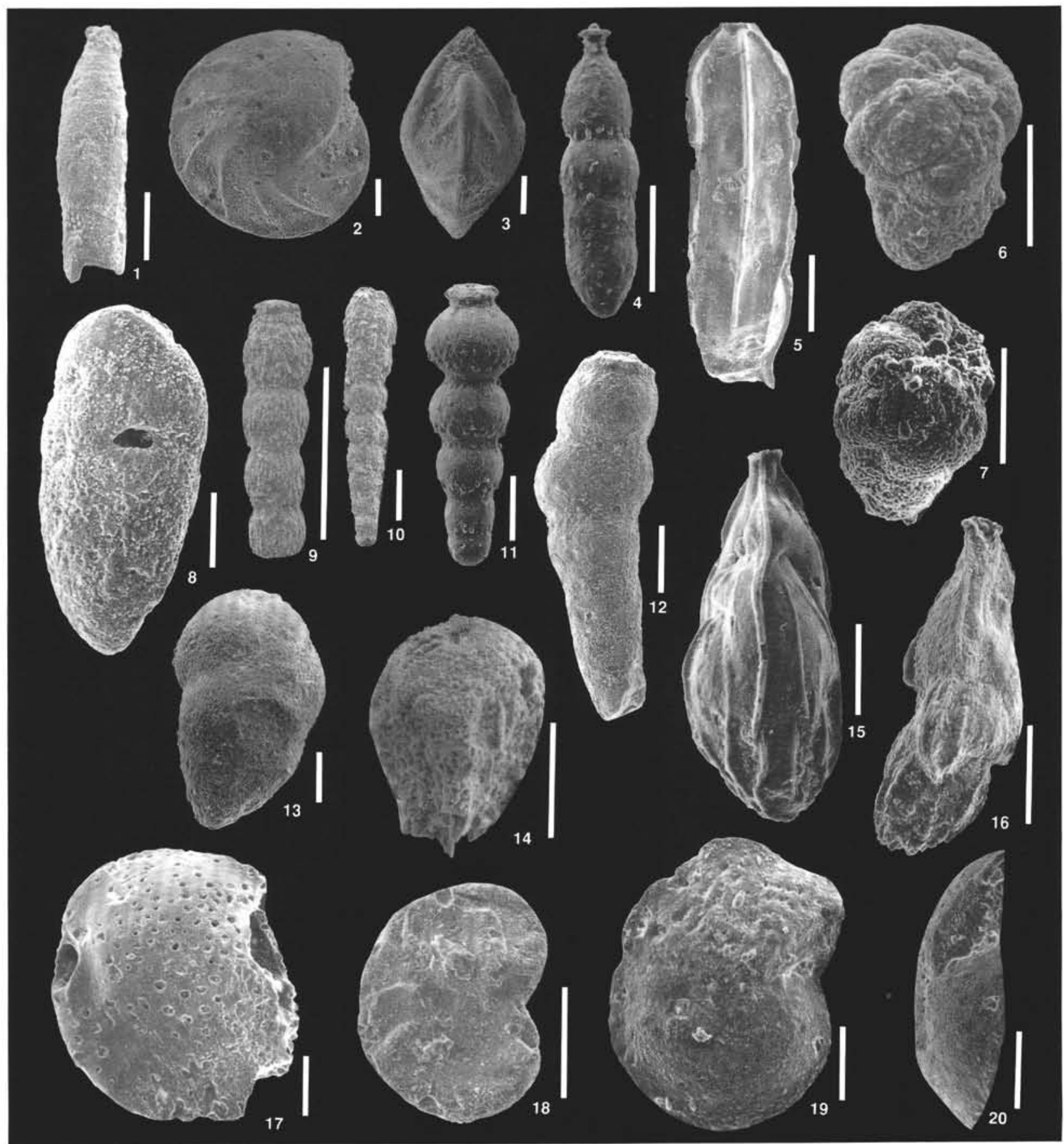

Plate 2. Scale bars $=100 \mu \mathrm{m}$, except $(5)$ and $(12)=300 \mu \mathrm{m}$. 1. Dentalina sp., Sample 129-802A-38R-4, 115-118 cm. 2, 3. Lenticulina sp., Sample 129-802A-27R-1, 124-129 cm. 4. Orphomorphina aff. antillea, Sample 129-802A-27R-1, 124-129 cm. 5. Orthomorphina cf. columnaris, Sample 129-802A$27 \mathrm{R}-1,124-129 \mathrm{~cm}$. 6, 7. Praebulimina cushmani, Sample 129-802A-38R-5, 54-59 cm. 8. Bolivina sp., Sample 129-802A-27R-1, 22-29 cm. 9, 10. Stilostomella pseudoscripta, Sample 129-802A-38R-4, 115-118 cm. 11. Stilostomella lepidula, Sample 129-802A-27R-1, 124-129 cm. 12. Stilostomella sp., Sample 129-802A-27R-1, 124-129 cm. 13. Bulimina tuxpamensis, Sample 129-802A-27R-1,22-29 cm. 14. Reussella cf. pseudospinulosa, Sample 129-802A38R-5, 54-59 cm. 15, 16. Angulogerina angulosa, Sample 129-802A-28R-3, 77-87 cm (15), Sample 129-802A-27R-1, 124-129 cm (16). 17. Siphonina cf. tenuicarinata, Sample 129-802A-28R-2, 40-46 cm. 18. Planulina $\mathrm{cf}$. wuellerstorfi, Sample 129-802A-38R-4, 115-118 cm. 19, 20. Cibicides ribbingi, Sample $129-802 \mathrm{~A}-38 \mathrm{R}-4,115-118 \mathrm{~cm}$. 


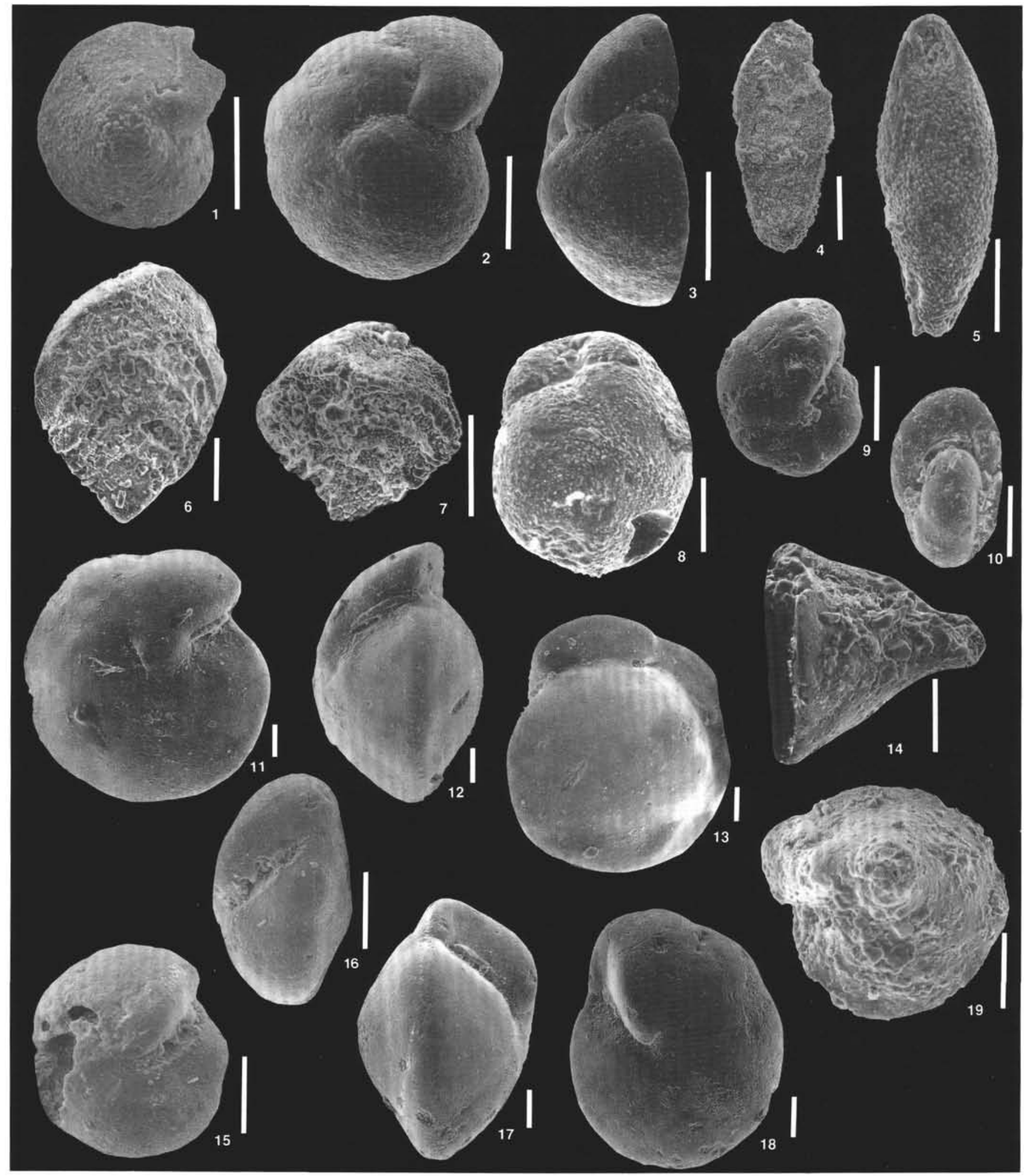

Plate 3. Scale bars $=100 \mu \mathrm{m} . \quad 1,2,3$. Cibicides voltziana, Sample 129-802A-31R-CC (1), Sample 129-802A-27R-1, 22-29 cm (2) and (3), same specimen. 4. Loxoxtomum eleyi, Sample 129-802A-38R-4, 115-118 cm. 5. Pleurostomella $\mathrm{cf}$. alternans, Sample 129-802A-28R-3, 77-87 cm. 6. Aragonia velascoensis, Sample 129-802A-38R-4, 115-118 cm. 7. Aragonia ouezzaensis, Sample 129-802A-38R-5, 54-59 cm. 8. Cassidulina subtumida, Sample 129-802A-28R-2, 40-46 cm. 9, 10. Pullenia minuta, Sample 129-802A-38R-4, 115-118 cm. 11, 12, 13. Oridorsalis umbonatus, Sample 129-802A-27R-1, 124-129 cm. 14, 19. Globorotalites michelianus, Sample 129-802A-38R-4, 115-118 cm. 15, 16. Gyroidinoides beisseli, Sample 129-802A-38R-4, 115-118 cm. 17, 18. Gyroidinoides cf. praeglobosus, Sample 129-802A-27R-1, 124-129 cm. 


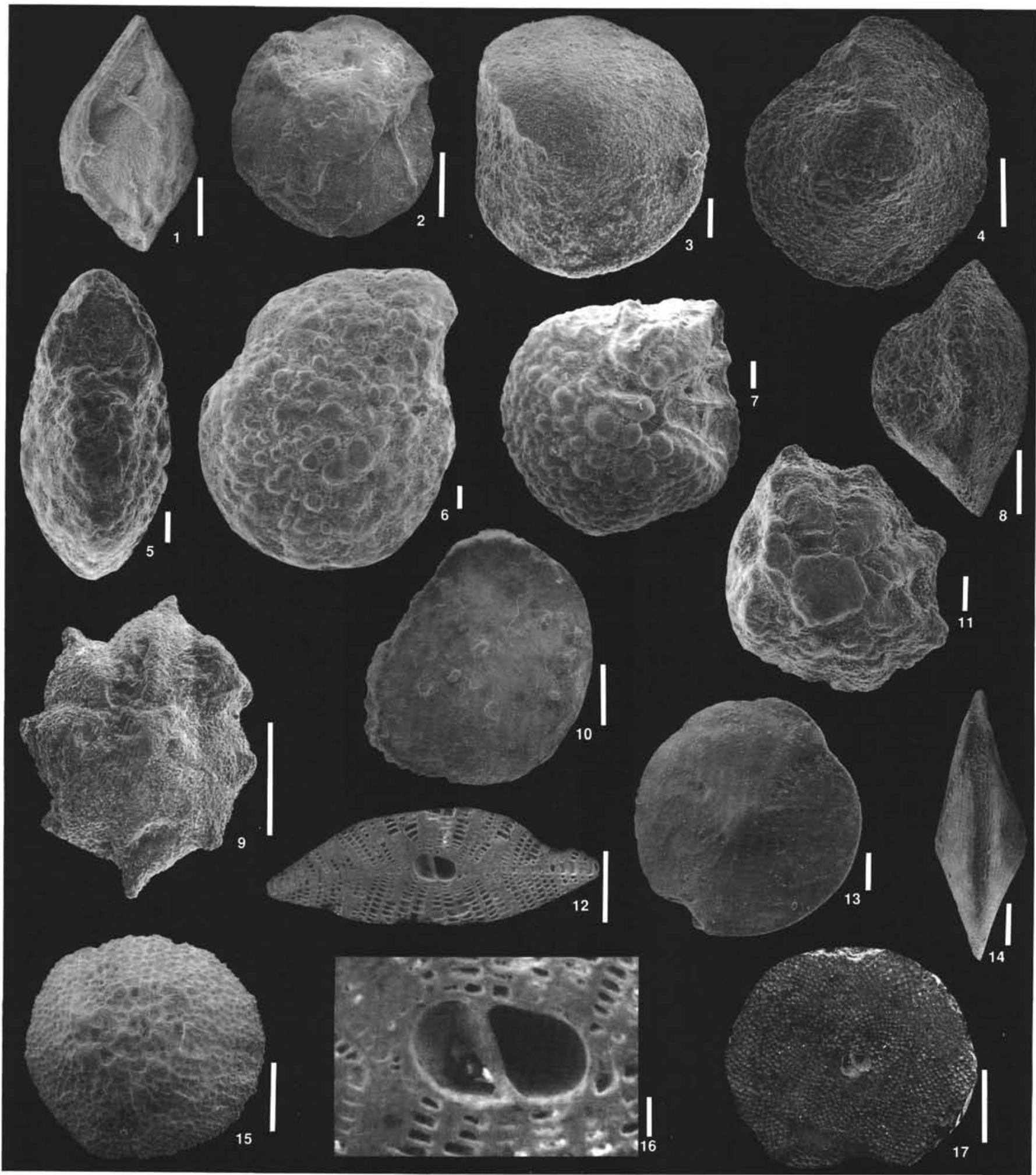

Plate 4. Scale bars $=100 \mu \mathrm{m}$, except (10), (12), (15), and $(17)=1 \mathrm{~mm}$. 1, 2. Epistomina cf. caracolla, Sample 129-802A-38R-4, 115-118 cm. 3. Asterigerina marshallana, Sample 129-802A-28R-CC. 4, 8. Asterigerina tentoria, Sample 129-802A-28R-2, 40-46 cm. 5-7. Rotalia tectoria, Sample 129-802A-27R-2, 29-34 cm. 9, 11. Pararotalia armata, Sample 129-802A-27R-2, 124-129 cm (9), Sample 129-802A-28R-CC (11). 10. Heterostegina borneensis, Sample 129-802A-28R-3, 77-83 cm. 13,14. Amphistegina hauerina, Sample 129-802A-28R-CC. 12, 15, 16, 17. Lepidocyclina sumatrensis, Sample 129-802A-28R-3, $77-83 \mathrm{~cm}$, (12) axial section; (15) exterior of test; (16) enlargement of embryonic chambers in (12); (17) equatorial section. 


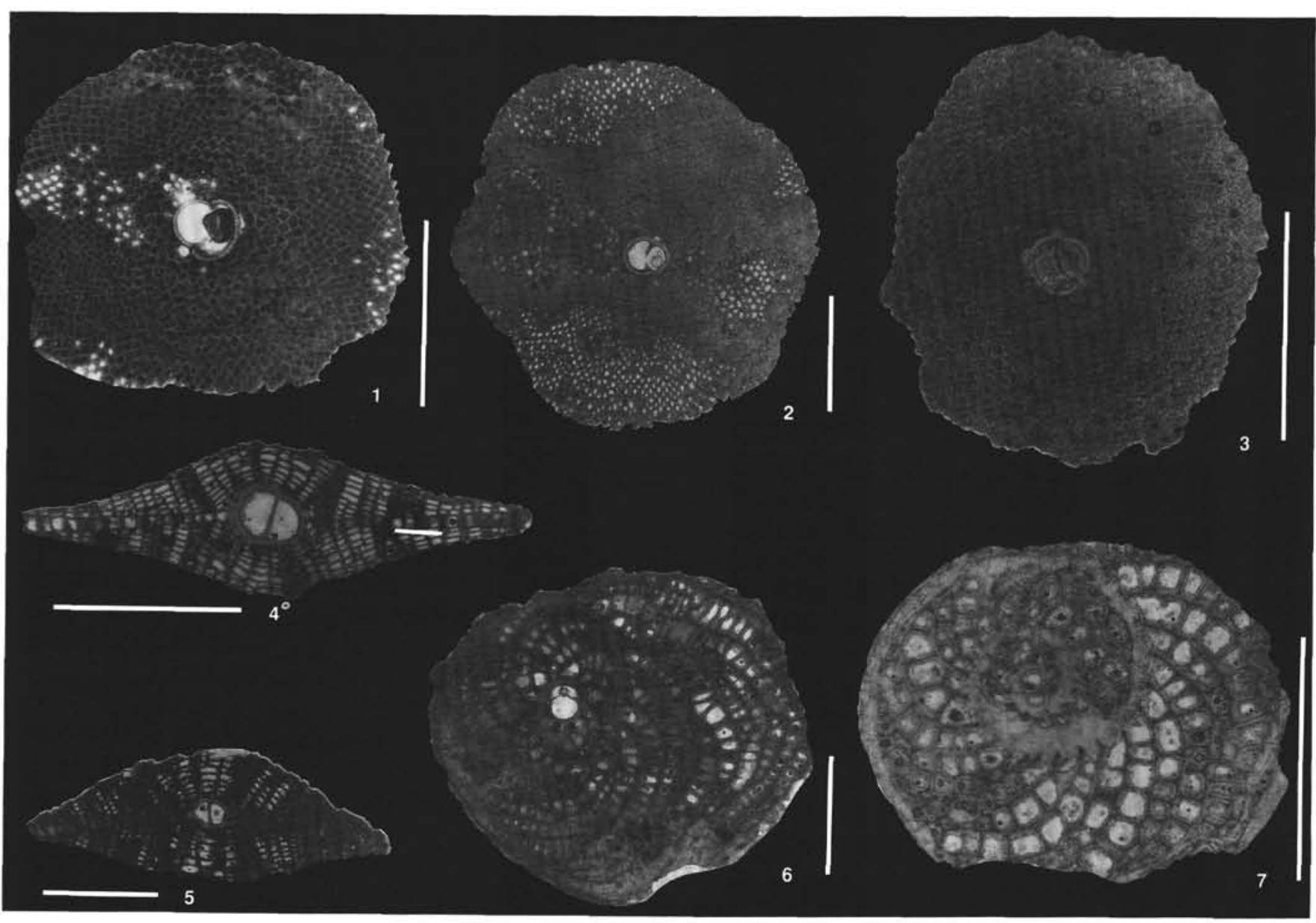

Plate 5. Scale bars $=1 \mathrm{~mm}$. 1-5. Lepidocyclina sumatrensis. Equatorial thin sections: (1) Sample 129-802A-28R-CC; (2) Sample 129-802A-28R-CC; (3) Sample 129-802A-27R-2, 29-34 cm. Axial thin sections: (4) Sample 129-802A-28R-CC; (5) Sample 129-802A-27R-1, 22-29 cm. 6, 7. Heterostegina borneensis. Equatorial thin sections: (6) Sample 129-802A-27R-2, 29-34 cm; (7) Sample 129-802A-27R-1, 22-29 cm. 